\title{
An Inverse Source Problem for Singular Parabolic Equations with Interior Degeneracy
}

\author{
Khalid Atifi $\left(\mathbb{D},{ }^{1}\right.$ Idriss Boutaayamou, ${ }^{2}$ Hamed Ould Sidi, ${ }^{1}$ and Jawad Salhi ${ }^{1}{ }^{1}$ \\ ${ }^{1}$ Faculté des Sciences et Techniques, Université Hassan 1er, Laboratoire MISI, BP 577, Settat 26000, Morocco \\ ${ }^{2}$ Faculté Polydisciplinaire de Ouarzazate, Université Ibn Zohr, BP 638, Ouarzazate 45000, Morocco \\ Correspondence should be addressed to Jawad Salhi; sj.salhi@gmail.com
}

Received 23 August 2018; Accepted 28 November 2018; Published 9 December 2018

Academic Editor: Changbum Chun

Copyright (c) 2018 Khalid Atifi et al. This is an open access article distributed under the Creative Commons Attribution License, which permits unrestricted use, distribution, and reproduction in any medium, provided the original work is properly cited.

\begin{abstract}
The main purpose of this work is to study an inverse source problem for degenerate/singular parabolic equations with degeneracy and singularity occurring in the interior of the spatial domain. Using Carleman estimates, we prove a Lipschitz stability estimate for the source term provided that additional measurement data are given on a suitable interior subdomain. For the numerical solution, the reconstruction is formulated as a minimization problem using the output least squares approach with the Tikhonov regularization. The Fréchet differentiability of the Tikhonov functional and the Lipschitz continuity of the Fréchet gradient are proved. These properties allow us to apply gradient methods for numerical solution of the considered inverse source problem.
\end{abstract}

\section{Introduction}

Inverse problems appear in a wide range of scientific applications, such as geophysics, biological and medical imaging, material and structure characterization, electrical, mechanical and civil engineering, and finances. The resolution of inverse problems consists of estimating the parameters of the observed system or structure from available data of solutions. The unknown quantities are diverse, according to the inverse problems and phenomena studied, but typical unknowns are spatially varying coefficients and source terms.

In the present paper, we study the inverse problem of determining the source term in a degenerate heat equation perturbed by a singular potential from the theoretical analysis and numerical computation angles. More precisely, we consider the following problem:

$$
\begin{aligned}
u_{t}-\left(a u_{x}\right)_{x}-\frac{\lambda}{b(x)} u & =h(t, x), \quad(t, x) \in Q, \\
u(0) & =u(1)=0, \quad t \in(0, T), \\
u(0, x) & =u_{0}(x), \quad x \in(0,1),
\end{aligned}
$$

where $u_{0} \in L^{2}(0,1), T>0$ fixed, and $Q:=(0, T) \times(0,1)$. Moreover, we assume that the constant $\lambda$ satisfies suitable assumptions described below and the functions $a$ and $b$ degenerate at the same interior point $x_{0}$ of the spatial domain $(0,1)$ (for the precise assumptions we refer to Section 2). Let us recall that, in inverse source problems, the source term has to satisfy some condition; otherwise uniqueness may be false; see [1]. Let $C_{0}>0$ be given and for $t_{0} \in(0, T)$ given, let $T^{\prime}:=\left(T+t_{0}\right) / 2$. In $[2,3]$, the authors make the assumption that source terms $h$ satisfy the condition

$$
\left|h_{t}(t, x)\right| \leq C_{0}\left|h\left(T^{\prime}, x\right)\right|, \quad \text { for almost all }(t, x) \in Q \text {. }
$$

Therefore they define the set $\delta\left(C_{0}\right)$ of admissible source terms as

$$
\mathcal{S}\left(C_{0}\right):=\left\{h \in H^{1}\left(0, T ; L^{2}(0,1)\right): h \text { satisfies }(2)\right\} .
$$

The Carleman estimate is a class of weighted energy estimates with a large parameter for a solution to a PDE and it is one of the major tools used in the study of unique continuation, observability, and controllability problems for various kinds of PDEs. The idea of using global Carleman estimates to solve inverse problems and prove Lipschitz stability results was first introduced by Puel and Yamamoto [4] in 1996 in the context of the wave equation, using a modification of the idea of [5]. Later on, it also has been 
applied to the standard heat equation by Imanuvilov and Yamamoto [2] in 1998. Their method is based on the use of global Carleman estimates for parabolic problems that were developed by Fursikov and Imanuvilov [6] and used to solve null controllability issues. The novelty of their work is that they not only solve the uniqueness question but they also provide unconditional Lipschitz stability result concerning the reconstruction of the source.

In the last recent years an increasing interest has been devoted to (1) in the case when $\lambda=0$ and the degeneracy can occur at the boundary or in the interior of the space domain. For example, we recall the works [7-9], where the authors obtain results concerning Carleman estimates and null controllability.

These results are complemented in $[3,10-12]$, where the authors obtain results concerning inverse problems for purely degenerate (i.e., $\lambda=0$ ) parabolic equations and parabolic coupled systems, addressing, in particular, issues such as uniqueness and stability. If $\lambda \neq 0$, the first results in this direction are obtained in [1] for the nondegenerate heat operator (i.e., $a>0$ ) with a singular potential. But, the study of numerical reconstruction questions are rarely taken into account; see $[13,14]$.

Furthermore, in both theoretical and numerical aspects, very few results are known regarding the identification of coefficients in degenerate/singular parabolic equations, even though this class of operators occurs in interesting theoretical and applied problems. As far as we know, [15] is the unique published work on this subject; it concerns the reconstruction of the initial heat distribution in a degenerate/singular parabolic equation with degeneracy and singularity at the boundary of the domain.

From the mathematical point of view and in connection with the work of Fragnelli and Mugnai (see [16]), we focus on identifying, on the basis of some observations, the source term, in a parabolic equation presenting both a degenerate diffusion coefficient and a singular potential with degeneracy and singularity inside the spatial domain.

In particular, our results complement the ones of $[1,3]$ in the purely degenerate case and in the purely singular one, respectively. More precisely, we will follow the approach introduced in [2] for the treatment of uniformly parabolic problems which is based on the use of global Carleman estimates. For this purpose, we use and extend some recent Carleman estimates for degenerate/singular equations obtained by Fragnelli and Mugnai [16]. As a consequence, we prove a stability estimate of Lipschitz type in determining the term source using the following observations data:

$$
\begin{gathered}
\left(\left(a u_{x}\right)_{x}+\frac{\lambda}{b} u\right)\left(T^{\prime}, \cdot\right), \\
\text { and }\left.u_{t}\right|_{\left(t_{0}, T\right) \times \omega},
\end{gathered}
$$

where the subregion of measurements $\omega$ is a nonempty subinterval of $(0,1)$ that is assumed to satisfy the following.

Hypothesis 1. The set of observation $\omega \subset \subset(0,1)$ is such that

$$
\omega=\omega_{1} \cup \omega_{2},
$$

where $\omega_{i} \subset(0,1), i=1,2$ are intervals with $\omega_{1} \subset \subset\left(0, x_{0}\right)$, $\omega_{2} \subset \subset\left(x_{0}, 1\right)$, and $x_{0} \notin \bar{\omega}$.

For fixed $T>T^{\prime}>0$, the main result of this paper can be stated as follows.

Theorem 2. Let $C_{0}>0$ and suppose that Hypotheses 1 and 14 are satisfied. Then, there exists $C=C\left(T, t_{0}, x_{0}, C_{0}\right)>0$ such that, for all $h \in S\left(C_{0}\right)$ and $u_{0} \in L^{2}(0,1)$,

$$
\begin{aligned}
& \|h\|_{L^{2}(\mathrm{Q})}^{2} \leq C\left[\left\|\left(a u_{x}\right)_{x}\left(T^{\prime}, \cdot\right)+\frac{\lambda}{b(\cdot)} u\left(T^{\prime}, \cdot\right)\right\|_{L^{2}(0,1)}^{2}\right. \\
& \left.+\left\|u_{t}\right\|_{L^{2}\left(\left(t_{0}, T\right) \times \omega\right)}^{2}\right] .
\end{aligned}
$$

\section{Remark 3.}

(i) It is worth noting that the result announced in Theorem 2 is still valid also in the case in which the observation set $\omega$ is an interval containing the degeneracy point. Indeed, if $x_{0} \in \omega$ one can always find two subintervals $\omega_{1} \subset\left(0, x_{0}\right), \omega_{2}=\subset\left(x_{0}, 1\right)$ such that $\left(\omega_{1} \cup \omega_{2}\right) \subset \subset \omega \backslash\left\{x_{0}\right\}$.

(ii) If we restrict ourselves to the particular case $h \in$ $\left\{r g, g \in L^{2}(0,1)\right\}$ for some given function $r \in$ $C^{1}([0, T] \times[0,1])$, positive at some time $t=T^{\prime}$ and $g$ is the unknown function that we want to recover, then uniqueness result can be shown as an immediate consequence of the Lipschitz stability result; see [17, Theorem 2.11].

In fact, we will not only investigate the theoretical aspect of the inverse source problem due to our interest in mathematics, but also consider the numerical reconstruction of the source term $h(t, x)$. To this end, we adopt the classical Tikhonov regularization to reformulate the inverse problem into a related optimization problem, for which we develop an iterative thresholding algorithm by using the corresponding adjoint system. In particular, we will focus on the determination of the unknown source term from the measured data at the final time. The resolution of this problem is standard and it is based on the gradient of the cost functional. More precisely, the most important issue in numerical solutions of inverse problems is the Lipschitz continuity of the Fréchet gradient. Indeed, in order to construct an effective minimization algorithm for an inverse problem, one needs to analyze the gradient of the considered cost functional. There is a vast literature on inverse problems for linear parabolic equations with final overdetermination. For example, we mention the pioneering work [18]. Compared to a standard parabolic equation, the main challenge here is the nonstandard degeneracy of the diffusion coefficient as well as the singularity of the potential of the partial differential equation (1).

The rest of this article is organized as follows. In Section 2, we recall the well-posedness of the problem (1). Then Section 3 is devoted to the proof of the main stability result of Lipschitz type. In Section 4, we reformulate our 
inverse source problem as a minimization problem with the Tikhonov regularization and provide a monotone iteration scheme based on a gradient method.

Throughout the paper, $C$ denotes a generic positive constant, which may vary from line to line.

\section{Well-Posedness}

The ways in which $a$ and $b$ degenerate at $x_{0}$ can be quite different, and for this reason, following [16], to establish our results, we give the following definitions and assumptions.

Hypothesis 4 (double weakly degenerate case (WWD)). There exists $x_{0} \in(0,1)$ such that $a\left(x_{0}\right)=b\left(x_{0}\right)=0, a, b>0$ in $[0,1] \backslash\left\{x_{0}\right\}, a, b \in C^{1}\left([0,1] \backslash\left\{x_{0}\right\}\right)$ and there exists $K, L \in(0,1)$ such that $\left(x-x_{0}\right) a^{\prime} \leq K a$ and $\left(x-x_{0}\right) b^{\prime} \leq L b$ a.e. in $[0,1]$.

Hypothesis 5 (weakly strongly degenerate case (WSD)). There exists $x_{0} \in(0,1)$ such that $a\left(x_{0}\right)=b\left(x_{0}\right)=0, a, b>0$ in $[0,1] \backslash\left\{x_{0}\right\}, a \in C^{1}\left([0,1] \backslash\left\{x_{0}\right\}\right), b \in C^{1}\left([0,1] \backslash\left\{x_{0}\right\}\right) \cap$ $W^{1, \infty}(0,1), \exists K \in(0,1), L \in[1,2)$ such that $\left(x-x_{0}\right) a^{\prime} \leq K a$ and $\left(x-x_{0}\right) b^{\prime} \leq L b$ a.e. in $[0,1]$.

Hypothesis 6 (strongly weakly degenerate case (SWD)). There exists $x_{0} \in(0,1)$ such that $a\left(x_{0}\right)=b\left(x_{0}\right)=0$, $a, b>0$ in $[0,1] \backslash\left\{x_{0}\right\}, a \in C^{1}\left([0,1] \backslash\left\{x_{0}\right\}\right) \cap W^{1, \infty}(0,1)$, $b \in C^{1}\left([0,1] \backslash\left\{x_{0}\right\}\right), \exists K \in[1,2), L \in(0,1)$ such that $\left(x-x_{0}\right) a^{\prime} \leq K a$ and $\left(x-x_{0}\right) b^{\prime} \leq L b$ a.e. in $[0,1]$.

Hypothesis 7 (double strongly degenerate case (SSD)). There exists $x_{0} \in(0,1)$ such that $a\left(x_{0}\right)=b\left(x_{0}\right)=0, a, b>0$ in $[0,1] \backslash\left\{x_{0}\right\}, a, b \in C^{1}\left([0,1] \backslash\left\{x_{0}\right\}\right) \cap W^{1, \infty}(0,1)$, there exists $K, L \in[1,2)$ such that $\left(x-x_{0}\right) a^{\prime} \leq K a$ and $\left(x-x_{0}\right) b^{\prime} \leq L b$ a.e. in $[0,1]$.

For the well-posedness of the problem (1), as in [16], we consider different classes of weighted Hilbert spaces, which are suitable to study the four different situations given above, namely, the (WWD), (WSD), (SWD), and (SSD) cases. Thus, we consider the Hilbert spaces

$$
H_{a}^{1}(0,1):=\left\{u \in W_{0}^{1,1}(0,1): \sqrt{a} u_{x} \in L^{2}(0,1)\right\}
$$

and

$$
H_{a, b}^{1}(0,1):=\left\{u \in H_{a}^{1}(0,1): \frac{u}{\sqrt{b}} \in L^{2}(0,1)\right\}
$$

endowed with the inner products

$$
\langle u, v\rangle_{H_{a}^{1}}:=\int_{0}^{1} a u^{\prime} v^{\prime} d x+\int_{0}^{1} u v d x,
$$

and

$$
\langle u, v\rangle_{H_{a, b}^{1}}:=\int_{0}^{1} a u^{\prime} v^{\prime} d x+\int_{0}^{1} u v d x+\int_{0}^{1} \frac{u v}{b} d x,
$$

respectively.

In order to deal with the singularity of $b$ we need the following inequality proved in [16, Proposition 2.14].
Lemma 8. If one among Hypotheses 4-6 holds with $K+L \leq 2$, then there exists a constant $C>0$ such that for all $u \in$ $H_{a, b}^{1}(0,1)$ we have

$$
\int_{0}^{1} \frac{u^{2}}{b(x)} d x \leq C \int_{0}^{1} a(x)\left|u^{\prime}\right|^{2} d x .
$$

In order to study well-posedness of problem (1) and in view of Lemma 8, we consider the space

$$
\mathscr{H}:=H_{a, b}^{1}(0,1),
$$

where the Hardy-Poincaré inequality (11) holds.

We underline that, from Lemma 8, the standard norm $\|$. $\|_{\mathscr{H}}^{2}$ is equivalent to

$$
\|\cdot\|_{\sim}^{2}:=\int_{0}^{1} a\left(u^{\prime}\right)^{2} d x .
$$

From now on, we make the following assumptions on $a$, $b$, and $\lambda$.

Hypothesis 9. (1) One among the Hypotheses 4, 5, or 6 holds true with $K+L \leq 2$ and we assume that

$$
\lambda \in\left(0, \frac{1}{C^{\star}}\right) .
$$

(2) Hypotheses 4, 5, 6, or 7 hold with $\lambda<0$.

Using Lemma 8, the next inequality is proved in $[16$, Proposition 2.18], which is crucial not only to obtain the wellposedness of problem (1), but also to prove that the inverse problem posed as weak solution minimization problem has a solution.

Proposition 10. Assume Hypothesis 9. Then there exists a positive constant $\Lambda \in(0,1]$ such that, for all $u \in \mathscr{H}$, there holds

$$
\int_{0}^{1} a\left(u^{\prime}\right)^{2} d x-\lambda \int_{0}^{1} \frac{u^{2}}{b} d x \geq \Lambda \int_{0}^{1} a\left(u^{\prime}\right)^{2} d x .
$$

Now, let us go back to problem (1), recalling the following definition.

Definition 11. Let $u_{0} \in L^{2}(0,1)$ and $h \in L^{2}(Q)$. A function $u$ is said to be a (weak) solution of (1) if

$$
u \in C\left([0, T] ; L^{2}(0,1)\right) \cap L^{2}(0, T ; \mathscr{H})
$$

and satisfies the following differential equation:

$$
\begin{aligned}
\int_{0}^{1} u(T, x) \varphi(T, x) d x-\int_{0}^{1} u_{0}(x) \varphi(0, x) d x \\
\quad-\iint_{Q} u(t, x) \varphi_{t}(t, x) d x d t \\
=-\iint_{Q} a(x) u_{x}(t, x) \varphi_{x}(t, x) d x d t \\
\quad+\lambda \iint_{Q} \frac{u(t, x) \varphi(t, x)}{b} d x d t \\
\quad+\iint_{Q} h(t, x) \varphi(t, x) d x d t
\end{aligned}
$$

for all $\varphi \in H^{1}\left(0, T ; L^{2}(0,1)\right) \cap L^{2}(0, T ; \mathscr{H})$. 
Finally, we introduce the Hilbert space

$$
\begin{aligned}
& H_{a, b}^{2}(0,1):=\left\{u \in H_{a}^{1}(0,1): a u^{\prime} \in H^{1}(0,1) \text { and } A u\right. \\
& \left.\quad \in L^{2}(0,1)\right\}
\end{aligned}
$$

where

$$
A u:=\left(a u^{\prime}\right)^{\prime}+\frac{\lambda}{b} u
$$

with domain

$$
D(A):=H_{a, b}^{2}(0,1) .
$$

Remark 12. Observe that if $u \in D(A)$, then $u / \sqrt{b} \in L^{2}(0,1)$, so that $u \in \mathscr{H}$ and inequality (11) holds.

Hence, the next result holds thanks to the theory of semigroups.

Proposition 13. The following assertions hold.

(i) The operator $(A, D(A))$ is the infinitesimal generator of a strongly continuous semigroup of contractions on $L^{2}(0,1)$. Moreover, the semigroup is analytic.

(ii) For all $u_{0} \in D(A)$ and $h \in H^{1}\left(0, T ; L^{2}(0,1)\right)$, problem (1) admits a unique strict solution belonging to the class

$$
u \in C([0, T] ; D(A)) \cap C^{1}\left([0, T] ; L^{2}(0,1)\right) .
$$

Moreover, if $u_{0} \in L^{2}(0,1)$, then for all $\varepsilon \in(0, T)$ there holds

$$
u \in C([\varepsilon, T] ; D(A)) \cap C^{1}\left([\varepsilon, T] ; L^{2}(0,1)\right) .
$$

(iii) For all $u_{0} \in L^{2}(0,1)$ and for all $h \in L^{2}\left(0, T ; L^{2}(0,1)\right)$, problem (1) has a unique weak solution $u \in C\left([0, T] ; L^{2}(0,1)\right) \cap$ $L^{2}(0, T ; \mathscr{H})$ such that for all $\varepsilon \in(0, T)$ there holds

$$
u \in L^{2}(\varepsilon, T ; D(A)) \cap H^{1}\left(\varepsilon, T ; L^{2}(0,1)\right) .
$$

Moreover, if $h \in H^{1}\left(0, T ; L^{2}(0,1)\right)$ and $\varepsilon \in(0, T)$, we have

$$
u \in H^{1}([\varepsilon, T] ; D(A)) \cap H^{2}\left([\varepsilon, T] ; L^{2}(0,1)\right)
$$

Proof. The proof of statement (i) can be found in [16], whereas statements (ii) and (iii) are a consequence of $(i)$ and [19, Proposition 3.3 and Proposition 3.8].

\section{Lipschitz Stability Result}

In this section, we aim at obtaining a Lipschitz stability result on determining the source term $h(x, t)$ in problem (1) in the spirit of the result by Imanuvilov and Yamamoto [2]. The key ingredient to obtain such a result is Carleman estimates. Here we use specific Carleman estimates for degenerate/singular parabolic equations (inspired by [16]). Thus, we first recall this fundamental tool in the following section before proving Theorem 2 in Section 3.2.
3.1. Carleman Estimate. The aim of this subsection is to prove a Carleman type inequality for solutions of problem (1). First of all, let us make precise the assumptions under which we consider problem (1).

Hypothesis 14. (1) Hypothesis 9 holds. Moreover, if $K>4 / 3$, then there exists $\vartheta \in(0, K]$ that the following condition is satisfied.

$(\mathscr{C})$ The function $x \longmapsto a /\left|x-x_{0}\right|^{9}$ is nonincreasing on the left of $x=x_{0}$ and nondecreasing on the right of $x=x_{0}$.

(2) Moreover, if $\lambda<0$, we require that

$$
\left(x-x_{0}\right) b^{\prime}(x) \geq 0 \text { in }[0,1] .
$$

As usual, the derivation of global Carleman estimates relies on the introduction of some suitable weight function of the form

$$
\varphi(t, x):=\theta(t) \psi(x), \quad \forall(t, x) \in\left(t_{0}, T\right) \times[-1,1],
$$

where

$$
\begin{gathered}
\theta(t):=\frac{1}{\left[\left(t-t_{0}\right)(T-t)\right]^{4}}, \\
\text { and } \psi(x):=c_{1}\left[\int_{x_{0}}^{x} \frac{y-x_{0}}{\tilde{a}(y)} d y-c_{2}\right],
\end{gathered}
$$

with $c_{2}>\widetilde{c}_{2}^{\star}:=\sup _{[-1,1]} \int_{x_{0}}^{x}\left(\left(y-x_{0}\right) / \widetilde{a}(y)\right) d y, c_{1}>0$, and where $\tilde{a}$ is defined by the following way:

$$
\tilde{a}(x):= \begin{cases}a(x), & x \in[0,1], \\ a(-x), & x \in[-1,0] .\end{cases}
$$

Observe that $\theta(t) \longrightarrow+\infty$ as $t \longrightarrow t_{0}^{+}, T^{-}$, and clearly

$$
-c_{1} c_{2} \leq \psi(x)<0 \text { for every } x \in[-1,1] \text {. }
$$

Eventually, we define as in [3] the second time weight function:

$$
\eta(t):=T+t_{0}-2 t, \quad \forall t \in\left(t_{0}, T\right) .
$$

Let us now turn to the following linear initial-boundary value problem:

$$
\begin{aligned}
\frac{\partial u}{\partial t}-\left(a u_{x}\right)_{x}-\frac{\lambda}{b(x)} u & =h(t, x), \\
\quad(t, x) \in\left(t_{0}, T\right) \times(0,1), & \\
u(0) & =u(1)=0, \quad t \in\left(t_{0}, T\right),
\end{aligned}
$$

where $h \in L^{2}\left(t_{0}, T, L^{2}(0,1)\right)$. In the following, we denote

$$
\begin{aligned}
Q_{t_{0}}^{T} & :=\left(t_{0}, T\right) \times(0,1) \\
\text { and } \omega_{t_{0}}^{T} & :=\left(t_{0}, T\right) \times \omega .
\end{aligned}
$$

Now we are ready to state global Carleman estimates with boundary observation for system (31). 
Theorem 15. Assume Hypothesis 14. Then, there exist two positive constants $C$ and $s_{0}$ such that the solution $u$ of (31) in $L^{2}\left(\varepsilon, T ; H_{a, b}^{2}\right) \cap H^{1}([\varepsilon, T], \mathscr{H})$ satisfies, for all $s \geq s_{0}$,

$$
\begin{aligned}
& \iint_{Q_{t_{0}}^{T}}\left(s \theta a(x) u_{x}^{2}+s^{3} \theta^{3} \frac{\left(x-x_{0}\right)^{2}}{a(x)} u^{2}+s \theta^{3 / 2}|\eta \psi| u^{2}\right. \\
& \left.\quad+\frac{1}{s \theta} u_{t}^{2}\right) e^{2 s \varphi} d x d t \leq C\left(\iint_{Q_{t_{0}}^{T}} h^{2} e^{2 s \varphi} d x d t\right. \\
& \left.\quad+s c_{1} \int_{t_{0}}^{T}\left[\theta a\left(x-x_{0}\right) u_{x}^{2} e^{2 s \varphi}\right]_{x=0}^{x=1} d t\right) .
\end{aligned}
$$

Some part of estimate (33) is already proved in [16] and, even if we refer to [20] a few times, our proof is quite selfcontained. In [16], the authors prove a Carleman inequality that estimates the integrals of $s \theta a(x) u_{x}^{2}$ and $s^{3} \theta^{3}((x-$ $\left.\left.x_{0}\right)^{2} / a(x)\right) u^{2}$ (that were sufficient for control purposes). For inverse problems, these estimates are not sufficient and one also needs some additional estimate of $u$ with a special weight and some estimate of the derivative term $u_{t}$ that we added here in the statement of Theorem 15. The proof is based on the methods developed in [3].

Proof. The proof of Theorem 15 relies on the change of variables $w=e^{s \varphi} u$ with $s>0$. Then, from (31), we obtain

$$
\begin{aligned}
& \underbrace{-\left(a w_{x}\right)_{x}-s \varphi_{t} w-s^{2} a \varphi_{x}^{2} w-\frac{\lambda}{b} w}_{L_{s}^{+} w} \\
& +\underbrace{w_{t}+2 s a \varphi_{x} w_{x}+s\left(a \varphi_{x}\right)_{x} w}_{L_{s}^{-} w}=\underbrace{h e^{s \varphi}}_{h_{s}} .
\end{aligned}
$$

Moreover, $w\left(t_{0}, x\right)=w(T, x)=0$. This property allows us to apply the Carleman estimates established in [16, Lemma 3.8] to $w$ with $Q_{t_{0}}^{T}$ in place of $(0, T) \times(0,1)$

$$
\begin{aligned}
& \left\|L_{s}^{+} w\right\|_{Q_{t_{0}}^{T}}^{2}+\left\|L_{s}^{-} w\right\|_{Q_{t_{0}}^{T}}^{2}+\iint_{Q_{t_{0}}^{T}}\left(s^{3} \theta^{3} \frac{\left(x-x_{0}\right)^{2}}{a(x)} w^{2}\right. \\
& \left.\quad+s \theta a(x) w_{x}^{2}\right) d x d t \\
& \quad \leq C\left(\iint_{Q_{t_{0}}^{T}} h^{2} e^{2 s \varphi} d x d t\right. \\
& \left.\quad+s c_{1} \int_{t_{0}}^{T}\left[\theta a\left(x-x_{0}\right) u_{x}^{2} e^{2 s \varphi}\right]_{x=0}^{x=1} d t\right) .
\end{aligned}
$$

The operators $L_{s}^{+}$and $L_{s}^{-}$are not exactly the ones of [16]. However, one can prove that the Carleman estimates do not change. Using the previous estimate, we aim at proving estimate (33) that concerns the variable $u$.
Step 1. Estimate of $\iint_{Q_{t_{0}}^{T}} s \theta a(x) u_{x}^{2} e^{2 s \varphi} d x d t$ and $\iint_{Q_{t_{0}}^{T}} s^{3} \theta^{3}((x-$ $\left.\left.x_{0}\right)^{2} / a(x)\right) u^{2} e^{2 s \varphi} d x d t$. Replacing $w$ by $e^{s \varphi} u$, we immediately get from (35)

$$
\begin{aligned}
& \iint_{Q_{t_{0}}^{T}} s^{3} \theta^{3} \frac{\left(x-x_{0}\right)^{2}}{a(x)} u^{2} e^{2 s \varphi} d x d t \\
& \quad \leq C\left(\iint_{Q_{t_{0}}^{T}} h^{2} e^{2 s \varphi} d x d t\right. \\
& \left.\quad+s c_{1} \int_{t_{0}}^{T}\left[\theta a\left(x-x_{0}\right) u_{x}^{2} e^{2 s \varphi}\right]_{x=0}^{x=1} d t\right) .
\end{aligned}
$$

Moreover, $w_{x}=s \varphi_{x} u e^{s \varphi}+u_{x} e^{s \varphi}$. Therefore,

$$
\begin{aligned}
& \iint_{Q_{t_{0}}^{T}} s \theta a u_{x}^{2} e^{2 s \varphi} d x d t \\
& \quad \leq 2 \iint_{Q_{t_{0}}^{T}} s \theta a w_{x}^{2} d x d t \\
& \quad+2 \iint_{Q_{t_{0}}^{T}} s^{3} \theta^{3} \frac{\left(x-x_{0}\right)^{2}}{a(x)} u^{2} e^{2 s \varphi} d x d t .
\end{aligned}
$$

In conclusion, thanks to (35)-(37), we get

$$
\begin{aligned}
& \iint_{Q_{t_{0}}^{T}}\left(s \theta a(x) u_{x}^{2}+s^{3} \theta^{3} \frac{\left(x-x_{0}\right)^{2}}{a(x)} u^{2}\right) e^{2 s \varphi} d x d t \\
& \quad \leq C\left(\iint_{Q_{t_{0}}^{T}} h^{2} e^{2 s \varphi} d x d t\right. \\
& \left.\quad+s c_{1} \int_{t_{0}}^{T}\left[\theta a\left(x-x_{0}\right) u_{x}^{2} e^{2 s \varphi}\right]_{x=0}^{x=1} d t\right) .
\end{aligned}
$$

Step 2. Estimate $\iint_{Q_{t_{0}}^{T}} s \theta^{3 / 2}|\eta \psi| u^{2} e^{2 s \varphi} d x d t$. Observe that, since $|\eta| \leq T-t_{0}$ and $|\psi| \leq c_{1} c_{2}$, one has

$$
\iint_{Q_{t_{0}}^{T}} s \theta^{3 / 2}|\eta \psi| w^{2} d x d t \leq C \iint_{Q_{t_{0}}^{T}} s \theta^{3 / 2} w^{2} d x d t .
$$

To estimate the integral on the right-hand side of (39), we follow the technique of [20, Lemma 4.1]. Using the Young inequality, we find

$$
\begin{aligned}
& \iint_{Q_{t_{0}}^{T}} s \theta^{3 / 2} w^{2} d x d t=s \iint_{Q_{t_{0}}^{T}}\left(\theta \frac{a^{1 / 3}}{\left|x-x_{0}\right|^{2 / 3}} w^{2}\right)^{3 / 4} \\
& \cdot\left(\theta^{3} \frac{\left|x-x_{0}\right|^{2}}{a} w^{2}\right)^{1 / 4} d x d t \leq s \frac{3}{4} \iint_{Q_{t_{0}}^{T}} \theta \\
& \cdot \frac{a^{1 / 3}}{\left|x-x_{0}\right|^{2 / 3}} w^{2} d x d t+s \frac{1}{4} \iint_{Q_{t_{0}}^{T}} \theta^{3} \\
& \quad \frac{\left|x-x_{0}\right|^{2}}{a} w^{2} d x d t .
\end{aligned}
$$


Now, if $K \leq 4 / 3$, we consider the function $p(x)=\mid x-$ $\left.x_{0}\right|^{4 / 3}$. Obviously, there exists $q \in(1,4 / 3)$ such that the function $p(x) /\left|x-x_{0}\right|^{q}$ is nonincreasing on the left of $x_{0}$ and nondecreasing on the right of $x_{0}$. Then, we can apply the Hardy-Poincaré inequality given in [20, Proposition 1.1], obtaining

$$
\begin{aligned}
& \int_{0}^{1} \frac{a^{1 / 3}}{\left|x-x_{0}\right|^{2 / 3}} w^{2} d x \\
& \quad \leq \max _{x \in[0,1]} a^{1 / 3}(x) \int_{0}^{1} \frac{1}{\left|x-x_{0}\right|^{2 / 3}} w^{2} d x \\
& \quad=\max _{x \in[0,1]} a^{1 / 3}(x) \int_{0}^{1} \frac{p(x)}{\left|x-x_{0}\right|^{2}} w^{2} d x \\
& \quad \leq \max _{x \in[0,1]} a^{1 / 3}(x) C_{H P} \int_{0}^{1} p(x) w_{x}^{2} d x \\
& \quad=\max _{x \in[0,1]} a^{1 / 3}(x) C_{H P} \int_{0}^{1} a \frac{\left|x-x_{0}\right|^{4 / 3}}{a} w_{x}^{2} d x \\
& \quad \leq \max _{x \in[0,1]} a^{1 / 3}(x) C_{H P} C_{1} \int_{0}^{1} a w_{x}^{2} d x,
\end{aligned}
$$

where $C_{H P}$ is the Hardy-Poincaré constant and $C_{1}=$ $\max \left(x_{0}^{4 / 3} / a(0),\left(1-x_{0}\right)^{4 / 3} / a(1)\right)$.

In the previous inequality, we have used the property that the map $x \longmapsto\left|x-x_{0}\right|^{\gamma} / a(x)$ is nonincreasing on the left of $x_{0}$ and nondecreasing on the right of $x_{0}$ for all $\gamma \geq K$; see [20, Lemma 2.1]. If $K>4 / 3$, we can consider the function $p(x)=\left(a(x)\left|x-x_{0}\right|^{4}\right)^{1 / 3}$. Then we have

$$
\begin{aligned}
\frac{a^{1 / 3}}{\left|x-x_{0}\right|^{2 / 3}}= & \frac{p(x)}{\left(x-x_{0}\right)^{2}}, \\
& p(x)=a(x)\left(\frac{\left(x-x_{0}\right)^{2}}{a(x)}\right)^{2 / 3} \leq C_{2} a(x),
\end{aligned}
$$

where

$$
C_{2}:=\max \left\{\left(\frac{x_{0}^{2}}{a(0)}\right)^{2 / 3},\left(\frac{\left(1-x_{0}\right)^{2}}{a(1)}\right)^{2 / 3}\right\} .
$$

Moreover, using the condition $(\mathscr{C})$ given in Hypothesis 14 , one has that the function $p(x) /\left|x-x_{0}\right|^{q}$, with $q:=(4+9) / 3 \epsilon$ $(1,2)$, is nonincreasing on the left of $x_{0}$ and nondecreasing on the right of $x_{0}$. The Hardy-Poincaré inequality given in [20, Proposition 1.1] implies

$$
\begin{aligned}
\int_{0}^{1} \frac{a^{1 / 3}}{\left|x-x_{0}\right|^{2 / 3}} w^{2} d x & =\int_{0}^{1} \frac{p}{\left(x-x_{0}\right)^{2}} w^{2} d x \\
& \leq C_{H P} \int_{0}^{1} p\left(w_{x}\right)^{2} d x \\
& \leq C_{H P} C_{2} \int_{0}^{1} a\left(w_{x}\right)^{2} d x .
\end{aligned}
$$

Thus, in every case, for $s$ large enough there holds

$$
\begin{aligned}
& \iint_{Q_{t_{0}}^{T}} s \theta^{3 / 2}|\eta \psi| w^{2} d x d t \\
& \quad \leq C \iint_{Q_{t_{0}}^{T}}\left(s \theta a w_{x}^{2}+s^{3} \theta^{3} \frac{\left|x-x_{0}\right|^{2}}{a} w^{2}\right) d x d t .
\end{aligned}
$$

Hence,

$$
\begin{aligned}
& \iint_{Q_{t_{0}}^{T}} s \theta^{3 / 2}|\eta \psi| w^{2} d x d t \leq C\left(\iint_{Q_{t_{0}}^{T}} h^{2} e^{2 s \varphi} d x d t\right. \\
& \left.\quad+s c_{1} \int_{t_{0}}^{T}\left[\theta a\left(x-x_{0}\right) u_{x}^{2} e^{2 s \varphi}\right]_{x=0}^{x=1} d t\right) .
\end{aligned}
$$

Finally, coming back to $u$, we get

$$
\begin{aligned}
& \iint_{Q_{t_{0}}^{T}} s \theta^{3 / 2}|\eta \psi| u^{2} e^{2 s \varphi} d x d t \leq C\left(\iint_{Q_{t_{0}}^{T}} h^{2} e^{2 s \varphi} d x d t\right. \\
& \left.\quad+s c_{1} \int_{t_{0}}^{T}\left[\theta a\left(x-x_{0}\right) u_{x}^{2} e^{2 s \varphi}\right]_{x=0}^{x=1} d t\right) .
\end{aligned}
$$

Step 3. Estimate of $\iint_{Q_{t_{0}}^{T}}(1 / s \theta) u_{t}^{2} e^{2 s \varphi} d x d t$. First of all, coming back to the definition of $L_{s}^{-} w$, we have

$$
\frac{1}{\sqrt{s \theta}} L_{s}^{-} w=\frac{1}{\sqrt{s \theta}} w_{t}+2 c_{1} \sqrt{s \theta}\left(x-x_{0}\right) w_{x}+c_{1} \sqrt{s \theta} w .
$$

Note that $1 / \sqrt{\theta}$ is bounded on $\left[t_{0}, T\right]$ and $\left|x-x_{0}\right|^{2} / a(x) \leq$ $\max \left\{x_{0}^{2} / a(0),\left(1-x_{0}\right)^{2} / a(1)\right\}$ (see [20, Lemma 2.1]). Therefore, there exists $C>0$ such that

$$
\begin{aligned}
& \iint_{Q_{t_{0}}^{T}} \frac{1}{s \theta} w_{t}^{2} d x d t \leq C\left(\left\|L_{s}^{-} w\right\|_{Q_{t_{0}}^{T}}^{2}\right. \\
& \left.\quad+\iint_{Q_{t_{0}}^{T}} s \theta \frac{\left|x-x_{0}\right|^{2}}{a} a w_{x}^{2} d x d t+\iint_{Q_{t_{0}}^{T}} s \theta w^{2} d x d t\right) \\
& \quad \leq C\left(\left\|L_{s}^{-} w\right\|_{Q_{t_{0}}^{T}}^{2}+\iint_{Q_{t_{0}}^{T}} s \theta a w_{x}^{2} d x d t\right. \\
& \left.\quad+\iint_{Q_{t_{0}}^{T}} s \theta w^{2} d x d t\right) .
\end{aligned}
$$

We then estimate $\iint_{Q_{t_{0}}^{T}} s \theta w^{2} d x d t$ thanks to Hardy inequality, as we have done in the previous step in (45). In this way, we find

$$
\begin{gathered}
\iint_{Q_{t_{0}}^{T}} s \theta w^{2} d x d t=s \iint_{Q_{t_{0}}^{T}}\left(\theta \frac{a^{1 / 3}}{\left|x-x_{0}\right|^{2 / 3}} w^{2}\right)^{3 / 4}(\theta \\
\left.. \frac{\left|x-x_{0}\right|^{2}}{a} w^{2}\right)^{1 / 4} d x d t \leq s \frac{3}{4} \iint_{Q_{t_{0}}^{T}} \theta
\end{gathered}
$$




$$
\begin{aligned}
& \cdot \frac{a^{1 / 3}}{\left|x-x_{0}\right|^{2 / 3}} w^{2} d x d t+\frac{s}{4} \iint_{Q_{t_{0}}^{T}} \theta \frac{\left|x-x_{0}\right|^{2}}{a} w^{2} d x d t \\
& \leq C \iint_{Q_{t_{0}}^{T}}\left(s \theta a w_{x}^{2}+s^{3} \theta^{3} \frac{\left(x-x_{0}\right)^{2}}{a} w^{2}\right) d x d t .
\end{aligned}
$$

Finally, using (49) and (35), one has

$$
\begin{gathered}
\iint_{Q_{t_{0}}^{T}} \frac{1}{s \theta} w_{t}^{2} d x d t \leq C\left(\iint_{Q_{t_{0}}^{T}} h^{2} e^{2 s \varphi} d x d t\right. \\
\left.\quad+s c_{1} \int_{t_{0}}^{T}\left[\theta a\left(x-x_{0}\right) u_{x}^{2} e^{2 s \varphi}\right]_{x=0}^{x=1} d t\right) .
\end{gathered}
$$

Now, in order to obtain the estimate of $u_{t}$ we have to use the estimate of (47). From the definition of $w$, we have $w_{t}=$ $u_{t} e^{s \varphi}+s \varphi_{t} w$. Hence

$$
\begin{aligned}
& \iint_{Q_{t_{0}}^{T}} \frac{1}{s \theta} u_{t}^{2} e^{2 s \varphi} d x d t \\
& \quad \leq 2\left(\iint_{Q_{t_{0}}^{T}} \frac{1}{s \theta} w_{t}^{2} d x d t+\iint_{Q_{t_{0}}^{T}} \frac{s^{2} \varphi_{t}^{2}}{s \theta} w^{2} d x d t\right) .
\end{aligned}
$$

The second term in the above right-hand side is estimated as follows:

$$
\begin{gathered}
\iint_{Q_{t_{0}}^{T}} \frac{s^{2} \varphi_{t}^{2}}{s \theta} w^{2} d x d t=16 \iint_{Q_{t_{0}}^{T}} s \theta^{3 / 2} \eta^{2} \psi^{2} w^{2} d x d t \\
\quad \leq 16\left(T-t_{0}\right)^{2} c_{1} c_{2} \iint_{Q_{t_{0}}^{T}} s \theta^{3 / 2}|\eta \psi| w^{2} d x d t .
\end{gathered}
$$

Hence using (47) and (51) we conclude that

$$
\begin{gathered}
\iint_{Q_{t_{0}}^{T}} \frac{1}{s \theta} u_{t}^{2} e^{2 s \varphi} d x d t \leq C\left(\iint_{Q_{t_{0}}^{T}} h^{2} e^{2 s \varphi} d x d t\right. \\
\left.\quad+s c_{1} \int_{t_{0}}^{T}\left[\theta a\left(x-x_{0}\right) u_{x}^{2} e^{2 s \varphi}\right]_{x=0}^{x=1} d t\right) .
\end{gathered}
$$

Conclusion. We immediately deduce the expected Carleman estimate (33) from (38), (47), and (54).

3.2. Proof of Lipschitz Stability. The object of this subsection is to prove Theorem 2 which recovers a source term $h$ from the measured final data and the partial knowledge of $u_{t}$ over the subdomain $\omega \subset(0,1)$. In proving these kinds of stability estimates, the global Carleman estimate obtained in Theorem 15 will play a crucial part along with certain energy estimates.

In order to obtain our main result, we need to define the following weights function associated with nondegenerate Carleman estimates in a general interval $(A, B)$ which are suited to our purpose. For $x \in[A, B]$,

$$
\begin{aligned}
\Phi(t, x) & =\theta(t) \Psi(x), \\
\Psi(x) & =e^{\rho \sigma(x)}-e^{2 \rho\|\sigma\|_{\infty},}
\end{aligned}
$$

where $\rho>0$ and $\sigma$ a $C^{2}([A, B])$ function such that $\sigma(x)>0$ in $(A, B), \sigma(A)=\sigma(B)=0$ and $\sigma_{x}(x) \neq 0$ in $[A, B] \backslash \widetilde{\omega}, \widetilde{\omega}$ is an open subset of $\omega$.

Now, choose the constant $c_{1}$ in (27) so that

$$
c_{1} \geq \frac{e^{2 \rho\|\sigma\|_{\infty}}-1}{c_{2}-\widetilde{c}_{2}^{\star}} .
$$

Thus, one can show that weight functions satisfy the following properties which are needed in the sequel.

Lemma 16. For $(t, x) \in[0, T] \times[0,1]$, we have

$$
\begin{aligned}
\varphi(t, x) & \leq \Phi(t, x) \text { and } \\
\varphi(t,-x) & \leq \Phi(t, x) .
\end{aligned}
$$

Proof. First, let us set $c_{2}^{\star}:=\sup _{[0,1]} \int_{x_{0}}^{x}\left(\left(y-x_{0}\right) / \widetilde{a}(y)\right) d y$.

(i) $\varphi \leq \Phi$. For all $x \in[0,1]$, we have

$$
\begin{array}{r}
-c_{1} c_{2} \leq \psi(x) \leq c_{1}\left(c_{2}^{\star}-c_{2}\right), \text { and } \\
1-e^{2 \rho\|\sigma\|_{\infty}} \leq \Psi(x) \leq e^{\rho\|\sigma\|_{\infty}}-e^{2 \rho\|\sigma\|_{\infty}} .
\end{array}
$$

Thus, to show that $\psi \leq \Psi$, it suffices to have

$$
c_{1}\left(c_{2}^{\star}-c_{2}\right) \leq 1-e^{2 \rho\|\sigma\|_{\infty}} .
$$

This means $c_{1} \geq\left(e^{2 \rho\|\sigma\|_{\infty}}-1\right) /\left(c_{2}-c_{2}^{\star}\right)$ and the conclusion follows immediately.

(ii) $\varphi(t,-x) \leq \Phi(t, x)$. In a similar manner, using (56), one has $\max \{\psi(-1), \psi(0)\} \leq \Psi(x)$ and the thesis follows.

Proof of Theorem 2. Let $u_{0} \in L^{2}(0,1), h \in S\left(C_{0}\right)$, and $t_{0} \in$ $(0, T)$. Observe that, according to Proposition 13 (iii), the solution $u$ of the problem (1) belongs to

$$
H^{1}\left(\left[t_{0}, T\right] ; D(A)\right) \cap H^{2}\left(\left[t_{0}, T\right] ; L^{2}(0,1)\right) .
$$

It follows that the solution $u$ of (1) satisfies sufficient regularity properties to proceed to the following computations. Let $z:=u_{t}$ where $u$ satisfies (1). Then $z \in L^{2}\left(\left[t_{0}, T\right] ; D(A)\right) \cap$ $H^{1}\left(\left[t_{0}, T\right] ; L^{2}(0,1)\right)$ and satisfies

$$
\begin{aligned}
z_{t}-\left(a z_{x}\right)_{x}-\frac{\lambda}{b(x)} z & =h_{t}(t, x), \quad(t, x) \in Q, \\
z(0) & =z(1)=0, \quad t \in(0, T), \\
z(0, x) & =\left(a u_{0 x}\right)_{x}+\frac{\lambda}{b(x)} u_{0}+h(0, x), \\
x & \in(0,1) .
\end{aligned}
$$

Let us recall that our goal is to provide an estimate of $\|h\|_{L^{2}(Q)}$. For this purpose, we divide the proof into four steps and we recall that $h \in H^{1}\left(t_{0}, T, L^{2}(0,1)\right)$.

Step 1 (Carleman estimates with locally distributed observation). Such estimates are obtained by studying some auxiliary 
problems, introduced by means of a suitable cut-off argument. First, by the assumption on the observation set, we can fix two subintervals $\omega_{1}:=\left(\alpha_{1}, \beta_{1}\right) \subset \subset\left(0, x_{0}\right), \omega_{2}:=$ $\left(\alpha_{2}, \beta_{2}\right) \subset \subset\left(x_{0}, 1\right)$ and four points $\gamma_{i}, \gamma_{i}^{\prime} \in\left(\alpha_{i}, \beta_{i}\right), i=1,2$, with $\gamma_{i}<\gamma_{i}^{\prime}$. Then, fix $\widetilde{\beta}_{2} \in\left(\alpha_{2}, \gamma_{2}\right)$ and consider a smooth function $\eta:[0,1] \longrightarrow[0,1]$ such that

$$
\eta(x)= \begin{cases}1, & x \in\left[\gamma_{2}, 1\right] \\ 0, & x \in\left[0, \widetilde{\beta}_{2}\right] .\end{cases}
$$

Let us now introduce $Z=\eta z$ where $z$ is the solution of (61). Hence, fixed $\widetilde{\alpha}_{2} \in\left(\alpha_{2}, \widetilde{\beta}_{2}\right) ; Z$ is a solution of the nondegenerate nonsingular parabolic equation

$$
\begin{aligned}
Z_{t}-\left(a Z_{x}\right)_{x}-\frac{\lambda}{b} Z & =H, \quad(t, x) \in\left(t_{0}, T\right) \times\left(\widetilde{\alpha}_{2}, 1\right), \\
Z\left(t, \widetilde{\alpha}_{2}\right) & =Z(t, 1)=0, \quad t \in\left(t_{0}, T\right),
\end{aligned}
$$

with $H:=\eta h_{t}-\left(a \eta_{x} z\right)_{x}-a \eta_{x} z_{x}$. Observe that, by the assumption on $a$ and the fact that $\eta_{x}$ is supported in $\breve{\omega}:=$ $\left(\widetilde{\beta}_{2}, \gamma_{2}\right) \subset \subset \omega_{2}$, we have $H \in L^{2}\left(\left(t_{0}, T\right) \times\left(\widetilde{\alpha}_{2}, 1\right)\right)$.

Thus, we can apply the analogue of [6, Lemma 1.2] for $Z$ in $\left(\widetilde{\alpha}_{2}, 1\right)$, obtaining that there exist two positive constants $C$ and $s_{0}\left(s_{0}\right.$ sufficiently large), such that $Z$ satisfies, for all $s \geq s_{0}$,

$$
\begin{aligned}
& \int_{t_{0}}^{T} \int_{\widetilde{\alpha}_{2}}^{1}\left(\frac{1}{s \theta} Z_{t}^{2}+s \theta Z_{x}^{2}+s^{3} \theta^{3} Z^{2}\right) e^{2 s \Phi} d x d t \\
& \quad \leq C\left(\int_{t_{0}}^{T} \int_{\widetilde{\alpha}_{2}}^{1} H^{2} e^{2 s \Phi} d x d t\right. \\
& \left.\quad+\iint_{\omega_{t_{0}}^{T}} s^{3} \theta^{3} Z^{2} e^{2 s \Phi} d x d t\right) .
\end{aligned}
$$

Using again the fact that $\eta_{x}$ is supported in $\breve{\omega}$ and the boundedness of $a$ (far away from $x_{0}$ in the weakly degenerate case and since $a \in W^{1, \infty}(0,1)$ in the strongly degenerate case), we have, by the Caccioppoli inequality for the nondegenerate case,

$$
\begin{aligned}
& \int_{t_{0}}^{T} \int_{\widetilde{\alpha}_{2}}^{1} H^{2} e^{2 s \Phi} d x d t \leq C \int_{t_{0}}^{T} \int_{\widetilde{\alpha}_{2}}^{1}\left(\eta^{2} h_{t}^{2}\right. \\
& \left.+\left(\left(a(x) \eta_{x} z\right)_{x}+a(x) \eta_{x} z_{x}\right)^{2}\right) e^{2 s \Phi} d x d t \\
& \quad \leq C\left(\int_{t_{0}}^{T} \int_{\widetilde{\alpha}_{2}}^{1} \eta^{2} h_{t}^{2} e^{2 s \Phi} d x d t+\iint_{\breve{\omega}_{t_{0}}}\left(z^{2}+z_{x}^{2}\right)\right. \\
& \left.\cdot e^{2 s \Phi} d x d t\right) \leq C\left(\int_{t_{0}}^{T} \int_{\widetilde{\alpha}_{2}}^{1} \eta^{2} h_{t}^{2} e^{2 s \Phi} d x d t\right. \\
& \left.+\iint_{\breve{\omega}_{t_{0}}}\left(h_{t}^{2}+s^{2} \theta^{2} z^{2}\right) e^{2 s \Phi} d x d t\right) .
\end{aligned}
$$

Hence, we can choose $s_{0}$ so large that for all $s \geq s_{0}$ and for a positive constant $C$, the following estimate holds:

$$
\begin{aligned}
& \int_{t_{0}}^{T} \int_{\widetilde{\alpha}_{2}}^{1}\left(\frac{1}{s \theta} Z_{t}^{2}+s \theta Z_{x}^{2}+s^{3} \theta^{3} Z^{2}\right) e^{2 s \Phi} d x d t \\
& \quad \leq C\left(\int_{t_{0}}^{T} \int_{\widetilde{\alpha}_{2}}^{1} \eta^{2} h_{t}^{2} e^{2 s \Phi} d x d t\right. \\
& \left.\quad+\iint_{\breve{\omega}_{t_{0}}}\left(h_{t}^{2}+s^{3} \theta^{3} z^{2}\right) e^{2 s \Phi} d x d t\right) .
\end{aligned}
$$

Furthermore, using the fact that $s \theta^{3 / 2}|\eta \psi| Z^{2} \leq C s^{3} \theta^{3} Z^{2}$, by (66) one has

$$
\begin{aligned}
& \int_{t_{0}}^{T} \int_{\widetilde{\alpha}_{2}}^{1} s \theta^{3 / 2}|\eta \psi| Z^{2} e^{2 s \Phi} d x d t \\
& \quad \leq C\left(\int_{t_{0}}^{T} \int_{\widetilde{\alpha}_{2}}^{1} \eta^{2} h_{t}^{2} e^{2 s \Phi} d x d t\right. \\
& \left.\quad+\iint_{\breve{\omega}_{t_{0}}}\left(h_{t}^{2}+s^{3} \theta^{3} z^{2}\right) e^{2 s \Phi} d x d t\right) .
\end{aligned}
$$

The estimates (66)-(67) lead to

$$
\begin{gathered}
\int_{t_{0}}^{T} \int_{\widetilde{\alpha}_{2}}^{1}\left(\frac{1}{s \theta} Z_{t}^{2}+s \theta^{3 / 2}|\eta \psi| Z^{2}+s \theta Z_{x}^{2}+s^{3} \theta^{3} Z^{2}\right) \\
\cdot e^{2 s \Phi} d x d t \leq C\left(\int_{t_{0}}^{T} \int_{\widetilde{\alpha}_{2}}^{1} \eta^{2} h_{t}^{2} e^{2 s \Phi} d x d t\right. \\
\left.+\iint_{\breve{\omega}_{t_{0}}}\left(h_{t}^{2}+s^{3} \theta^{3} z^{2}\right) e^{2 s \Phi} d x d t\right) .
\end{gathered}
$$

Now, since $x_{0} \notin\left(\widetilde{\alpha}_{2}, 1\right)$, by Lemma 16 we can prove that there exists a positive constant $k$ such that for every $(t, x) \in[0, T] \times$ $\left[\widetilde{\alpha}_{2}, 1\right]$

$$
\begin{aligned}
a(x) e^{2 s \varphi(t, x)} & \leq k e^{2 s \Phi} \text { and } \\
\frac{\left(x-x_{0}\right)^{2}}{a(x)} e^{2 s \varphi(t, x)} & \leq k e^{2 s \Phi} .
\end{aligned}
$$

Then, using (69), (68) becomes

$$
\begin{aligned}
& \int_{t_{0}}^{T} \int_{\widetilde{\alpha}_{2}}^{1}\left(\frac{1}{s \theta} Z_{t}^{2}+s \theta^{3 / 2}|\eta \psi| Z^{2}+s \theta a Z_{x}^{2}\right. \\
& \left.\quad+s^{3} \theta^{3} \frac{\left(x-x_{0}\right)^{2}}{a} Z^{2}\right) e^{2 s \varphi} d x d t \\
& \quad \leq k \int_{t_{0}}^{T} \int_{\widetilde{\alpha}_{2}}^{1}\left(\frac{1}{s \theta} Z_{t}^{2}+s \theta^{3 / 2}|\eta \psi| Z^{2}+s \theta Z_{x}^{2}\right. \\
& \left.+s^{3} \theta^{3} Z^{2}\right) e^{2 s \Phi} d x d t
\end{aligned}
$$




$$
\begin{aligned}
& \leq k C\left(\int_{t_{0}}^{T} \int_{\widetilde{\alpha}_{2}}^{1} \eta^{2} h_{t}^{2} e^{2 s \Phi} d x d t\right. \\
& \left.+\iint_{\breve{\omega}_{t_{0}}}\left(h_{t}^{2}+s^{3} \theta^{3} z^{2}\right) e^{2 s \Phi} d x d t\right) .
\end{aligned}
$$

Hence, using the definition of $Z$, it results

$$
\begin{aligned}
& \int_{t_{0}}^{T} \int_{\gamma_{2}}^{1}\left(\frac{1}{s \theta} z_{t}^{2}+s \theta^{3 / 2}|\eta \psi| z^{2}+s \theta a z_{x}^{2}\right. \\
& \left.+s^{3} \theta^{3} \frac{\left(x-x_{0}\right)^{2}}{a} z^{2}\right) e^{2 s \varphi} d x d t=\int_{t_{0}}^{T} \int_{\gamma_{2}}^{1}\left(\frac{1}{s \theta} Z_{t}^{2}\right. \\
& \left.+s \theta^{3 / 2}|\eta \psi| Z^{2}+s \theta a Z_{x}^{2}+s^{3} \theta^{3} \frac{\left(x-x_{0}\right)^{2}}{a} Z^{2}\right) \\
& \cdot e^{2 s \varphi} d x d t \leq \int_{t_{0}}^{T} \int_{\widetilde{\alpha}_{2}}^{1}\left(\frac{1}{s \theta} Z_{t}^{2}+s \theta^{3 / 2}|\eta \psi| Z^{2}\right. \\
& \left.+s \theta a Z_{x}^{2}+s^{3} \theta^{3} \frac{\left(x-x_{0}\right)^{2}}{a} Z^{2}\right) e^{2 s \varphi} d x d t \\
& \leq C\left(\iint_{Q_{t_{0}}^{T}} h_{t}^{2} e^{2 s \Phi} d x d t+\iint_{\omega_{t_{0}}^{T}}\left(h_{t}^{2}+s^{3} \theta^{3} z^{2}\right)\right. \\
& \left.\cdot e^{2 s \Phi} d x d t\right) \leq C\left(\iint_{Q_{t_{0}}^{T}} h_{t}^{2} e^{2 s \Phi} d x d t\right. \\
& \left.+\iint_{\omega_{t_{0}}^{T}} s^{3} \theta^{3} z^{2} e^{2 s \Phi} d x d t\right)
\end{aligned}
$$

for a positive constant $C$ and for $s$ large enough.

To complete the proof, it is sufficient to prove a similar inequality on the interval $\left[0, \gamma_{2}\right]$. To this aim, we follow a reflection procedure. Consider the function

$$
w(t, x):= \begin{cases}z(t, x), & x \in[0,1], \\ -z(t,-x), & x \in[-1,0] .\end{cases}
$$

Therefore, $w$ solves the problem

$$
\begin{aligned}
w_{t}-\left(\widetilde{a} w_{x}\right)_{x}-\frac{\lambda}{\widetilde{b}} w+ & =G, \quad(t, x) \in(0, T) \times(-1,1), \\
w(t,-1) & =w(t, 1)=0, \quad t \in(0, T),
\end{aligned}
$$

being

$$
\begin{gathered}
\tilde{b}(x):= \begin{cases}b(x), & x \in[0,1], \\
b(-x), & x \in[-1,0],\end{cases} \\
G= \begin{cases}h_{t}(x), & x \in[0,1], \\
h_{t}(-x), & x \in[-1,0] .\end{cases}
\end{gathered}
$$

Now, consider a smooth function $\tau:[-1,1] \longrightarrow[0,1]$ such that

$$
\tau(x)= \begin{cases}1, & x \in\left[-\gamma_{1}, \gamma_{2}\right] \\ 0, & x \in\left[-1,-\gamma_{1}^{\prime}\right] \cup\left[\gamma_{2}^{\prime}, 1\right]\end{cases}
$$

and define the function $W=\tau w$, where $w$ is the solution of (73). Then $W$ solves

$$
\begin{gathered}
W_{t}-\left(\widetilde{a} W_{x}\right)_{x}-\frac{\lambda}{\widetilde{b}} W=\tau G-\left(\tilde{a} \tau_{x} w\right)_{x}-\tilde{a} \tau_{x} w_{x}:=F, \\
(t, x) \in(0, T) \times\left(-\beta_{1}, 1\right), \\
W\left(t,-\beta_{1}\right)=W(t, 1)=0, \quad t \in(0, T) .
\end{gathered}
$$

Observe that $W_{x}\left(t,-\beta_{1}\right)=W_{x}(t, 1)=0$ and, by the assumption on $a$ and the fact that $\tau_{x}$ is supported in $\left[-\gamma_{1}^{\prime},-\gamma_{1}\right] \cup$ $\left[\gamma_{2}, \gamma_{2}^{\prime}\right], F \in L^{2}((0, T) \times I)$, where $I:=\left(-\beta_{1}, 1\right)$. Thus, we can apply the analogue of Theorem 15 (which still holds true, since $\widetilde{a}$ belongs to $W^{1,1}(-1,1)$ in the weakly degenerate case and to $W^{1, \infty}(-1,1)$ in the strongly degenerate one) on $\left(-\beta_{1}, 1\right)$ in place of $(0,1)$, obtaining that there exist two positive constants $C$ and $s_{0}$ ( $s_{0}$ sufficiently large), such that $W$ satisfies, for all $s \geq s_{0}$,

$$
\begin{gathered}
\int_{t_{0}}^{T} \int_{-\beta_{1}}^{1}\left[s \theta \tilde{a} W_{x}^{2}+s^{3} \theta^{3} \frac{\left(x-x_{0}\right)^{2}}{\tilde{a}} W^{2}+s \theta^{3 / 2}|\eta \psi| W^{2}\right. \\
\left.+\frac{1}{s \theta} W_{t}^{2}\right] e^{2 s \varphi} d x d t \leq C \int_{t_{0}}^{T} \int_{-\beta_{1}}^{1} F^{2} e^{2 s \varphi} d x d t .
\end{gathered}
$$

Using again the fact that $\tau_{x}$ is supported in $\left[-\gamma_{1}^{\prime},-\gamma_{1}\right] \cup$ $\left[\gamma_{2}, \gamma_{2}^{\prime}\right]$ and the boundedness of $\tilde{a}$ and $\tilde{a}^{\prime}$ (recall that, using the assumption on $a, \tilde{a}$ is $C^{1}$ far away from $x_{0}$ and 0 in the weakly degenerate case and it is $W^{1, \infty}(-1,1)$ in the strongly degenerate one), it follows that

$$
\begin{aligned}
& \int_{t_{0}}^{T} \int_{-\beta_{1}}^{1}\left[s \theta \tilde{a} W_{x}^{2}+s^{3} \theta^{3} \frac{\left(x-x_{0}\right)^{2}}{\widetilde{a}} W^{2}+s \theta^{3 / 2}|\eta \psi| W^{2}\right. \\
& \left.\quad+\frac{1}{s \theta} W_{t}^{2}\right] e^{2 s \varphi} d x d t \\
& \quad \leq C\left(\int_{t_{0}}^{T} \int_{-\beta_{1}}^{1} \tau^{2} G^{2} e^{2 s \varphi} d x d t\right. \\
& \left.\quad+\int_{t_{0}}^{T}\left(\int_{-\gamma_{1}^{\prime}}^{-\gamma_{1}}+\int_{\gamma_{2}}^{\gamma_{2}^{\prime}}\right)\left[w^{2}+w_{x}^{2}\right] e^{2 s \varphi} d x d t\right) .
\end{aligned}
$$

Now, by the definitions of $w$, we note that

$$
\begin{aligned}
& \int_{t_{0}}^{T} \int_{-\gamma_{1}^{\prime}}^{-\gamma_{1}}\left[w^{2}+w_{x}^{2}\right] e^{2 s \varphi(x)} d x d t \\
& \quad=\int_{t_{0}}^{T} \int_{-\gamma_{1}^{\prime}}^{-\gamma_{1}}\left[z^{2}(-x)+z_{x}^{2}(-x)\right] e^{2 s \varphi(x)} d x d t .
\end{aligned}
$$


Using a change of variable, by (57), one has

$$
\begin{aligned}
& \int_{t_{0}}^{T} \int_{-\gamma_{1}^{\prime}}^{-\gamma_{1}}\left[z^{2}(-x)+z_{x}^{2}(-x)\right] e^{2 s \varphi(x)} d x d t \\
& \quad=\int_{t_{0}}^{T} \int_{\gamma_{1}}^{\gamma_{1}^{\prime}}\left[z^{2}(x)+z_{x}^{2}(x)\right] e^{2 s \varphi(-x)} d x d t \\
& \quad \leq \int_{t_{0}}^{T} \int_{\gamma_{1}}^{\gamma_{1}^{\prime}}\left[z^{2}(x)+z_{x}^{2}(x)\right] e^{2 s \Phi(x)} d x d t .
\end{aligned}
$$

Consequently,

$$
\begin{aligned}
& \int_{t_{0}}^{T} \int_{-\gamma_{1}^{\prime}}^{-\gamma_{1}}\left[w^{2}+w_{x}^{2}\right] e^{2 s \varphi(x)} d x d t \\
& \quad \leq \int_{t_{0}}^{T} \int_{\gamma_{1}}^{\gamma_{1}^{\prime}}\left[z^{2}+z_{x}^{2}\right] e^{2 s \Phi(x)} d x d t .
\end{aligned}
$$

Going back to (78), by (81), and using the fact that $\varphi \leq \Phi$, we obtain

$$
\begin{aligned}
& \int_{t_{0}}^{T} \int_{-\beta_{1}}^{1}\left[s \theta \tilde{a} W_{x}^{2}+s^{3} \theta^{3} \frac{\left(x-x_{0}\right)^{2}}{\tilde{a}} W^{2}+s \theta^{3 / 2}|\eta \psi| W^{2}\right. \\
& \left.\quad+\frac{1}{s \theta} W_{t}^{2}\right] e^{2 s \varphi} d x d t \\
& \quad \leq C\left(\int_{t_{0}}^{T} \int_{-\beta_{1}}^{1} \tau^{2} G^{2} e^{2 s \Phi} d x d t\right. \\
& \left.\quad+\int_{t_{0}}^{T}\left(\int_{\gamma_{1}}^{\gamma_{1}^{\prime}}+\int_{\gamma_{2}}^{\gamma_{2}^{\prime}}\right)\left[z^{2}+z_{x}^{2}\right] e^{2 s \Phi} d x d t\right) .
\end{aligned}
$$

Thus, applying the Caccioppoli inequality and recalling that $G$ is defined through $h_{t}$, one can find $C>0$ such that

$$
\begin{aligned}
& \int_{t_{0}}^{T} \int_{-\beta_{1}}^{1}\left[s \theta \tilde{a} W_{x}^{2}+s^{3} \theta^{3} \frac{\left(x-x_{0}\right)^{2}}{\tilde{a}} W^{2}+s \theta^{3 / 2}|\eta \psi| W^{2}\right. \\
& \left.\quad+\frac{1}{s \theta} W_{t}^{2}\right] e^{2 s \varphi} d x d t \\
& \quad \leq C\left(\int_{t_{0}}^{T} \int_{-\beta_{1}}^{1} \tau^{2} G^{2} e^{2 s \Phi} d x d t\right. \\
& \quad+\int_{t_{0}}^{T}\left(\int_{\gamma_{1}}^{\gamma_{1}^{\prime}}+\int_{\gamma_{2}}^{\gamma_{2}^{\prime}}\right) z^{2} e^{2 s \Phi} d x d t \\
& \left.\quad+\int_{t_{0}}^{T}\left(\int_{\omega_{1}}+\int_{\omega_{2}}\right)\left[h_{t}^{2}+s^{2} \theta^{2} z^{2}\right] e^{2 s \Phi} d x d t\right) \\
& \quad \leq C\left(\iint_{Q_{t_{0}}^{T}} h_{t}^{2} e^{2 s \Phi} d x d t\right. \\
& \left.\quad+\int_{t_{0}}^{T}\left(\int_{\omega_{1}}+\int_{\omega_{2}}\right)\left[h_{t}^{2}+s^{2} \theta^{2} z^{2}\right] e^{2 s \Phi} d x d t\right) \\
& \quad \leq C\left(\iint_{Q_{t_{0}}^{T}} h_{t}^{2} e^{2 s \Phi} d x d t+\iint_{\omega_{t_{0}}^{T}} s^{2} \theta^{2} z^{2} e^{2 s \Phi} d x d t\right) .
\end{aligned}
$$

Hence, using the definitions of $w$ and $W$, there exists a constant $C>0$ such that

$$
\begin{aligned}
& \int_{t_{0}}^{T} \int_{0}^{\gamma_{2}}\left[s \theta a z_{x}^{2}+s^{3} \theta^{3} \frac{\left(x-x_{0}\right)^{2}}{a} z^{2}+s \theta^{3 / 2}|\eta \psi| z^{2}\right. \\
& \left.+\frac{1}{s \theta} z_{t}^{2}\right] e^{2 s \varphi} d x d t=\int_{t_{0}}^{T} \int_{0}^{\gamma_{2}}\left[s \theta \widetilde{a} w_{x}^{2}\right. \\
& \left.+s^{3} \theta^{3} \frac{\left(x-x_{0}\right)^{2}}{\tilde{a}} w^{2}+s \theta^{3 / 2}|\eta \psi| w^{2}+\frac{1}{s \theta} w_{t}^{2}\right] \\
& \cdot e^{2 s \varphi} d x d t=\int_{t_{0}}^{T} \int_{0}^{\gamma_{2}}\left[s \theta \widetilde{a} W_{x}^{2}\right. \\
& \left.+s^{3} \theta^{3} \frac{\left(x-x_{0}\right)^{2}}{\widetilde{a}} W^{2}+s \theta^{3 / 2}|\eta \psi| W^{2}+\frac{1}{s \theta} W_{t}^{2}\right] \\
& \cdot e^{2 s \varphi} d x d t \leq \int_{t_{0}}^{T} \int_{-\beta_{1}}^{1}\left[s \theta \widetilde{a} W_{x}^{2}\right. \\
& \left.+s^{3} \theta^{3} \frac{\left(x-x_{0}\right)^{2}}{\tilde{a}} W^{2}+s \theta^{3 / 2}|\eta \psi| W^{2}+\frac{1}{s \theta} W_{t}^{2}\right] \\
& \cdot e^{2 s \varphi} d x d t \leq C\left(\iint_{Q_{t_{0}}^{T}} h_{t}^{2} e^{2 s \Phi} d x d t\right. \\
& \left.+\iint_{\omega_{t_{0}}^{T}} s^{2} \theta^{2} z^{2} e^{2 s \Phi} d x d t\right) .
\end{aligned}
$$

Finally adding up (71) and (84), we conclude that

$$
\begin{aligned}
I_{0} & :=\iint_{Q_{t_{0}}^{T}}\left[s \theta a z_{x}^{2}+s^{3} \theta^{3} \frac{\left(x-x_{0}\right)^{2}}{a} z^{2}+s \theta^{3 / 2}|\eta \psi| z^{2}\right. \\
& \left.+\frac{1}{s \theta} z_{t}^{2}\right] e^{2 s \varphi} d x d t \\
& \leq C \underbrace{\left(\iint_{Q_{t_{0}}^{T}} h_{t}^{2} e^{2 s \Phi} d x d t+\iint_{\omega_{t_{0}}^{T}} s^{3} \theta^{3} z^{2} e^{2 s \Phi} d x d t\right)}_{I_{1}},
\end{aligned}
$$

for all $s \geq s_{0}$ and for a positive constant $C$.

Step 2 (estimate from above of $I_{1}$ ). In this step, our purpose is to show that there exists some constant $C>0$ such that

$$
I_{1} \leq C\left[\frac{1}{\sqrt{s}} \int_{0}^{1} h^{2}\left(T^{\prime}, x\right) e^{2 s \Phi\left(T^{\prime}, x\right)} d x+\left\|u_{t}\right\|_{L^{2}\left(\omega_{t_{0}}^{T}\right)}^{2}\right] .
$$

Let us recall that, by the definition of $\Psi$, there exists some constant $p<0$ such that $\Psi(x) \leq p$ for all $x \in[0,1]$. Therefore we have

$$
s^{3} \theta^{3} e^{2 s \Phi} \leq s^{3} \theta^{3} e^{2 s p \theta} \longrightarrow 0 \text { as } t \longrightarrow t_{0} \text { or } T .
$$


As a consequence, setting $C=\sup _{\mathbb{R}}\left\{y \longmapsto y^{3} e^{2 s p y}\right\}>0$, we have

$$
\iint_{\omega_{t_{0}}^{T}} s^{3} \theta^{3} z^{2} e^{2 s \Phi} d x d t \leq C\|z\|_{L^{2}\left(\omega_{t_{0}}^{T}\right)}^{2}=\left\|u_{t}\right\|_{L^{2}\left(\omega_{t_{0}}^{T}\right)}^{2} .
$$

In order to complete the proof of (86), it remains to prove the following lemma.

Lemma 17. There exists a constant $C>0$ such that

$$
\iint_{\mathbb{Q}_{t_{0}}^{T}} h_{t}^{2} e^{2 s \Phi} d x d t \leq \frac{C}{\sqrt{s}} \int_{0}^{1} h^{2}\left(T^{\prime}, x\right) e^{2 s \Phi\left(T^{\prime}, x\right)} d x .
$$

We omit the proof of Lemma 17 which is classical and we refer the reader to [2]. Using (88) and (89), we obtain (86).

Step 3 (estimate from below of $I_{0}$ ). The purpose of the step is to provide the following estimate: there exists a constant $C>0$ such that

$$
\int_{0}^{1} z^{2}\left(T^{\prime}, x\right) e^{2 s \varphi\left(T^{\prime}, x\right)} d x \leq C I_{0}
$$

Since $\lim _{t \rightarrow t_{0}} z^{2}(t, x) e^{2 s \varphi(t, x)}=0$, for a.e. $x \in(0,1)$, we can write

$$
\begin{aligned}
\int_{0}^{1} & z^{2}\left(T^{\prime}, x\right) e^{2 s \varphi\left(T^{\prime}, x\right)} d x \\
\quad= & \int_{t_{0}}^{T^{\prime}} \frac{\partial}{\partial t}\left(\int_{0}^{1} z^{2}(t, x) e^{2 s \varphi(t, x)} d x\right) d t \\
& =\int_{t_{0}}^{T^{\prime}} \int_{0}^{1}\left(2 z z_{t}+2 s \varphi_{t} z^{2}\right) e^{2 s \varphi(t, x)} d x d t .
\end{aligned}
$$

Using Young's inequality, one has

$$
\begin{aligned}
& \int_{t_{0}}^{T^{\prime}} \int_{0}^{1} 2 z z_{t} e^{2 s \varphi} d x d t \\
& \quad=\int_{t_{0}}^{T^{\prime}} \int_{0}^{1}\left(2 \sqrt{s \theta} z e^{s \varphi}\right)\left(\frac{1}{\sqrt{s \theta}} z_{t} e^{s \varphi}\right) d x d t \\
& \quad \leq \iint_{Q_{t_{0}}^{T}}\left(s \theta z^{2} e^{2 s \varphi}+\frac{1}{s \theta} z_{t}^{2} e^{2 s \varphi}\right) d x d t \\
& \quad \leq \iint_{Q_{t_{0}}^{T}} s \theta z^{2} e^{2 s \varphi} d x d t+I_{0} .
\end{aligned}
$$

Moreover, by the Hardy-Poincaré inequality, and using the same computation as in the proof of (45), we get

$$
\begin{aligned}
& \int_{t_{0}}^{T^{\prime}} \int_{0}^{1} 2 z z_{t} e^{2 s \varphi} d x d t \\
& \quad \leq C\left(\iint_{Q_{t_{0}}^{T}}\left(s \theta a(x) z_{x}^{2}+s^{3} \theta^{3} \frac{\left(x-x_{0}\right)^{2}}{a(x)} z^{2}\right)\right. \\
& \left.\quad \cdot e^{2 s \varphi} d x d t+I_{0}\right) \leq C I_{0} .
\end{aligned}
$$

Next, using the fact that $\left|\varphi_{t}\right| \leq C|\eta \psi| \theta^{3 / 2}$, we have

$$
\begin{aligned}
& \int_{t_{0}}^{T^{\prime}} \int_{0}^{1} s \varphi_{t} z^{2} e^{2 s \varphi} d x d t \\
& \quad \leq C \iint_{Q_{t_{0}}^{T}} s \theta^{3 / 2}|\eta \psi| z^{2} e^{2 s \varphi} d x d t \leq C I_{0} .
\end{aligned}
$$

Eventually, (91) associated with (92) and (93) gives (90).

Step 4 (conclusion). Using (90), (85), and next (86), there exists some constant $C>0$ such that

$$
\begin{aligned}
& \int_{0}^{1} z^{2}\left(T^{\prime}, x\right) e^{2 s \varphi\left(T^{\prime}, x\right)} d x d t \\
& \quad \leq C\left[\frac{1}{\sqrt{s}} \int_{0}^{1} h^{2}\left(T^{\prime}, x\right) e^{2 s \Phi\left(T^{\prime}, x\right)} d x+\left\|u_{t}\right\|_{L^{2}\left(\omega_{t_{0}}^{T}\right)}^{2}\right] .
\end{aligned}
$$

On the other hand, let us recall that $z$ satisfies

$$
\begin{aligned}
z\left(T^{\prime}, x\right) & =u_{t}\left(T^{\prime}, x\right) \\
& =\left(a u_{x}\right)_{x}\left(T^{\prime}, x\right)+\frac{\lambda}{b} u\left(T^{\prime}, x\right)+h\left(T^{\prime}, x\right)
\end{aligned}
$$

for a.e. $x \in[0,1]$.

Therefore,

$$
\begin{aligned}
& \int_{0}^{1} h^{2}\left(T^{\prime}, x\right) e^{2 s \varphi\left(T^{\prime}, x\right)} d x \\
& \quad \leq C\left(\left\|\left(a u_{x}\right)_{x}\left(T^{\prime}, x\right)+\frac{\lambda}{b} u\left(T^{\prime}, x\right)\right\|_{L^{2}(0,1)}^{2}\right. \\
& \left.\quad+\int_{0}^{1} z^{2}\left(T^{\prime}, x\right) e^{2 s \varphi\left(T^{\prime}, x\right)} d x\right) .
\end{aligned}
$$

Applying (95) to estimate the term $\int_{0}^{1} z^{2}\left(T^{\prime}, x\right) e^{2 s \varphi\left(T^{\prime}, x\right)} d x$, we obtain

$$
\begin{aligned}
& \int_{0}^{1} h^{2}\left(T^{\prime}, x\right) e^{2 s \varphi\left(T^{\prime}, x\right)} d x \\
& \quad \leq C\left(\left\|\left(a u_{x}\right)_{x}\left(T^{\prime}, x\right)+\frac{\lambda}{b} u\left(T^{\prime}, x\right)\right\|_{L^{2}(0,1)}^{2}\right. \\
& \left.\quad+\left\|u_{t}\right\|_{L^{2}\left(\omega_{t_{0}}^{T}\right)}^{2}+\frac{1}{\sqrt{s}} \int_{0}^{1} h^{2}\left(T^{\prime}, x\right) e^{2 s \Phi\left(T^{\prime}, x\right)} d x\right) .
\end{aligned}
$$

Choosing $s$ large enough such that $C / \sqrt{s}=1 / 2$, we get

$$
\begin{aligned}
& \int_{0}^{1} h^{2}\left(T^{\prime}, x\right) e^{2 s \varphi\left(T^{\prime}, x\right)} d x \\
& \quad \leq C\left[\left\|\left(a u_{x}\right)_{x}\left(T^{\prime}, x\right)+\frac{\lambda}{b} u\left(T^{\prime}, x\right)\right\|_{L^{2}(0,1)}^{2}\right. \\
& \left.\quad+\left\|u_{t}\right\|_{L^{2}\left(\omega_{t_{0}}^{T}\right)}^{2}\right] .
\end{aligned}
$$


On the other hand, $s$ being now fixed, there exists some constant $\gamma>0$ such that

$$
e^{2 s \varphi\left(T^{\prime}, x\right)} \geq \gamma>0
$$

So we can write

$$
\begin{aligned}
& \gamma \int_{0}^{1} h^{2}\left(T^{\prime}, x\right) d x \\
& \quad \leq C\left[\left\|\left(a u_{x}\right)_{x}\left(T^{\prime}, x\right)+\frac{\lambda}{b} u\left(T^{\prime}, x\right)\right\|_{L^{2}(0,1)}^{2}\right. \\
& \left.\quad+\left\|u_{t}\right\|_{L^{2}\left(\omega_{t_{0}}^{T}\right)}^{2}\right] .
\end{aligned}
$$

Moreover, in view of (2),

$$
\begin{aligned}
|h(t, x)| & \leq\left|h\left(T^{\prime}, x\right)\right|+\int_{T^{\prime}}^{t}\left|h_{t}(s, x)\right| d s \\
& \leq C\left|h\left(T^{\prime}, x\right)\right| .
\end{aligned}
$$

Hence,

$$
\begin{aligned}
& \|h\|_{L^{2}(\mathrm{Q})}^{2} \leq C \iint_{Q} h^{2}\left(T^{\prime}, x\right) d x d t \\
& \quad=C T \int_{0}^{1} h^{2}\left(T^{\prime}, x\right) d x \\
& \quad \leq C\left[\left\|\left(a u_{x}\right)_{x}\left(T^{\prime}, x\right)+\frac{\lambda}{b} u\left(T^{\prime}, x\right)\right\|_{L^{2}(0,1)}^{2}\right. \\
& \left.\quad+\left\|u_{t}\right\|_{L^{2}\left(\omega_{t_{0}}^{T}\right)}^{2}\right]
\end{aligned}
$$

which concludes the proof.

\section{Numerical Approach}

In this section, we develop an algorithm for numerically reconstructing the unknown source term from the measured final data.

4.1. Solvability of the Inverse Problem and Gradient Formula. As the theoretical stability is guaranteed by Theorem 2, in this subsection we study the inverse source problem from the numerical viewpoint. To this end, let us define our inverse problem which we use in computations.

Inverse Source Problem (ISP). Let $u$ be the solution to (1). Determine the source term $h(t, x)$ from the measured data at the final time $u(T,$.$) .$

Problem (ISP) will be defined as the inverse source problem associated with the singular parabolic equation (1). Accordingly, for a given function $h \in \mathcal{U}$, from some class of admissible source functions $\mathcal{U}$, which will be defined below, the singular parabolic problem (1) will be referred to as a direct problem. A solution of the direct problem (1), corresponding to the function $h \in \mathcal{U}$, will be defined as $u(t, x ; h)$.
Remark 18. It should be mentioned that we do not need the supplement distributed measurements to obtain the numerical solution of the inverse problem.

Numerically, we treat ISP by interpreting its solution as a minimizer of the following least squares functional with the Tikhonov regularization:

$$
\begin{aligned}
& \min _{h \in \mathscr{U}} J(h), \\
& J(h)=\frac{1}{2}\|u(T, x ; h)-\widetilde{u}(x)\|_{L^{2}(0,1)}^{2}+\frac{\varepsilon}{2}\left\|h-h_{0}\right\|_{L^{2}(Q)}^{2},
\end{aligned}
$$

where $\tilde{u} \in L^{2}(0,1)$ is the observation data with noise, $h_{0}$ is the initial guess for $h, \varepsilon>0$ stands for the regularization parameter, and $\mathcal{U}$ is the set of admissible unknown sources defined in the following way:

$$
\begin{aligned}
\mathscr{U} & :=\left\{h \in H^{1}\left(0, T ; L^{2}(0,1)\right):\|h\|_{H^{1}\left(0, T ; L^{2}(0,1)\right)}\right. \\
& \leq r, r>0\} .
\end{aligned}
$$

Evidently, the set $\mathcal{U}$ is a bounded, closed, and convex subset of $H^{1}\left(0, T ; L^{2}(0,1)\right)$.

We are now going to show the existence of minimizers to problem (104). To do so, we need the following lemma.

Lemma 19. Assume Hypothesis 9. Let $u$ be the weak solution of (1) corresponding to a given source term $h$. Then, the function $F: H^{1}\left(0, T ; L^{2}(0,1)\right) \longrightarrow C\left([0, T] ; L^{2}(0,1)\right) \cap L^{2}(0, T ; \mathscr{H})$ defined as $F(h):=u$ is continuous.

Proof. First, take $u_{0} \in D(A)$. Then, let the source term $h$ be perturbed by a small amount $\delta h$ such that $h+\delta h \in \mathcal{U}$. Consider $\delta u=u^{\delta}-u$, where $u^{\delta}$ is the weak solution of (1) with source term $h^{\delta}:=h+\delta h$. Then $\delta u \in C^{1}\left([0, T] ; L^{2}(0,1)\right) \cap$ $C(0, T ; D(A))$ satisfies the following sensitivity problem:

$$
\begin{aligned}
\partial_{t} \delta u-\partial_{x}\left(a \partial_{x} \delta u\right)-\frac{\lambda}{b} \delta u & =\delta h(t, x), \quad(t, x) \in Q, \\
\delta u(0) & =\delta u(1)=0, \quad t \in(0, T), \\
\delta u(0, x) & =0, \quad x \in(0,1) .
\end{aligned}
$$

Let $v(t, x)$ be a smooth function. From (106) and by the Gauss Green identity [16, Lemma 2.21], we have

$$
\begin{gathered}
\int_{0}^{1} \partial_{t} \delta u v d x+\int_{0}^{1}\left(a \partial_{x} \delta u v_{x}-\frac{\lambda}{b} \delta u v\right) d x \\
\quad=\int_{0}^{1} \delta h v d x .
\end{gathered}
$$

We take $\delta u$ as a mutual test function for $v$ to deduce

$$
\begin{aligned}
& \frac{1}{2} \frac{d}{d t} \int_{0}^{1}(\delta u)^{2} d x+\int_{0}^{1}\left(a\left(\partial_{x} \delta u\right)^{2}-\frac{\lambda}{b}(\delta u)^{2}\right) d x \\
& \quad=\int_{0}^{1} \delta h \delta u d x
\end{aligned}
$$


Then, using Lemma 8, by the Cauchy-Schwarz inequality we obtain

$$
\begin{gathered}
\frac{1}{2} \frac{d}{d t}\|\delta u(t)\|_{L^{2}(0,1)}^{2}+\Lambda \int_{0}^{1} a\left(\partial_{x} \delta u(t)\right)^{2} d x \\
\leq \frac{1}{2}\|\delta u(t)\|_{L^{2}(0,1)}^{2}+\frac{1}{2}\|\delta h(t)\|_{L^{2}(0,1)}^{2},
\end{gathered}
$$

for every $t \leq T$, from which

$$
\frac{d}{d t}\|\delta u(t)\|_{L^{2}(0,1)}^{2} \leq\|\delta u(t)\|_{L^{2}(0,1)}^{2}+\|\delta h(t)\|_{L^{2}(0,1)}^{2} .
$$

Applying Gronwall's inequality, we obtain

$$
\begin{aligned}
\|\delta u(t)\|_{L^{2}(0,1)}^{2} & \leq e^{T}\left(\|\delta u(0)\|_{L^{2}(0,1)}^{2}+\|\delta h\|_{L^{2}(\mathrm{Q})}^{2}\right) \\
& =e^{T}\|\delta h\|_{L^{2}(\mathrm{Q})}^{2}
\end{aligned}
$$

for every $t \leq T$. From (109) and (111), we immediately get

$$
\int_{0}^{T}\left\|\sqrt{a} \delta u_{x}(t)\right\|_{L^{2}(0,1)}^{2} d t \leq C_{T}\|\delta h\|_{L^{2}(Q)}^{2},
$$

for every $t \leq T$ and some universal constant $C_{T}>0$. Thus, by (111) and (112), we obtain

$$
\begin{aligned}
& \sup _{t \in[0, T]}\|\delta u(t)\|_{L^{2}(0,1)}^{2}+\int_{0}^{T}\|\delta u(t)\|_{\mathscr{H}}^{2} d t \\
& \quad \leq C_{T}\|\delta h\|_{L^{2}(\mathrm{Q})}^{2},
\end{aligned}
$$

from which it follows that

$$
\begin{aligned}
& \|\delta u\|_{C\left([0, T] ; L^{2}(0,1)\right)}^{2}+\|\delta u\|_{L^{2}(0, T ; \mathscr{H})}^{2} \\
& \leq C\|\delta h\|_{H^{1}\left(0, T ; L^{2}(0,1)\right)}^{2},
\end{aligned}
$$

if $u_{0} \in D(A)$. Since $D(A)$ is dense in $L^{2}(0,1)$, the same inequality holds if $u_{0} \in L^{2}(0,1)$. This completes the proof Lemma 19.

An immediate consequence of Lemma 19 is the following result.

Proposition 20. Assume Hypothesis 9. Then, the functional J is continuous on $\mathcal{U}$ and there exists a minimizer $h^{\star} \in \mathcal{U}$ of $J(h)$, i.e.,

$$
J\left(h^{\star}\right)=\min _{h \in \mathscr{U}} J(h) .
$$

Here we will propose an iterative method to solve the nonlinear optimization problem (104), and thus the information of the derivatives of the objective functional plays a significant and important role. Our approach for computing the Fréchet derivatives is based on adjoint state method. This used method is also called the variational adjoint method or the adjoint problem approach [18, 21, 22]. A distinct advantage of using such a method is due to the fact that it permits to reduce the computational costs as well as its relatively simple numerical implementation.

The following proposition characterizes the derivative of the cost functional (104).
Proposition 21. The Tikhonov functional J is Fréchet differentiable and its derivative at each $h \in \mathcal{U}$ is given by

$$
J^{\prime}(h)=v+\varepsilon\left(h-h_{0}\right),
$$

where $v \in C\left([0, T] ; L^{2}(0,1)\right)$ is the mild solution of the following adjoint equation:

$$
\begin{aligned}
v_{t}+\left(a v_{x}\right)_{x}+\frac{\lambda}{b(x)} v & =0, \quad(t, x) \in Q, \\
v(0) & =v(1)=0, \quad t \in(0, T), \\
v(T, x) & =u(T)-\tilde{u}, \quad x \in(0,1) .
\end{aligned}
$$

For the proof of the above result, we shall use the following lemma which derives an integral relationship relating the change $\delta h$ in the term source to the change of the output $\delta u(T)$ through the solution of an adjoint problem.

Lemma 22. Let $h, h^{\delta}$ be given elements. Denote by $u$ and $u^{\delta}$ the solutions of the direct problem (1) corresponding to the given admissible term sources $h, h^{\delta} \in \mathcal{U}$. Then the following integral identity holds:

$$
\int_{0}^{1} \delta u(T) q(x) d x=\int_{0}^{T} \int_{0}^{1} \delta h v d x d t
$$

where $\delta u(T)=u^{\delta}(T)-u(T), \delta h=h^{\delta}-h$, and $v(t, x):=$ $v(t, x ; q)$ solves the adjoint problem

$$
\begin{aligned}
v_{t}+\left(a v_{x}\right)_{x}+\frac{\lambda}{b(x)} v & =0, \quad(t, x) \in Q, \\
v(0) & =v(1)=0, \quad t \in(0, T), \\
v(T, x) & =q(x), \quad x \in(0,1),
\end{aligned}
$$

with an arbitrary input $q(x) \in L^{2}(0,1)$.

Proof of Proposition 21. Let $u$ and $u^{\delta}$ be the solutions of the direct problem (1) corresponding the source terms $h, h^{\delta}=h+$ $\delta h \in \mathcal{U}$. By calculating the increment $\delta J(h):=J\left(h^{\delta}-J(h)\right)$ of the cost functional, we deduce that

$$
\begin{aligned}
\delta J(h)= & \int_{0}^{1}(u(T)-\tilde{u}) \delta u(T) d x+\frac{1}{2}\|\delta u(T)\|_{L^{2}(0,1)}^{2} \\
& +\varepsilon \int_{0}^{T} \int_{0}^{1}\left(h-h_{0}\right) \delta h d x d t+\frac{\varepsilon}{2}\|\delta h\|_{L^{2}(\mathrm{Q})}^{2},
\end{aligned}
$$

for all $h, h^{\delta} \in \mathcal{U}$, where $\delta u=u^{\delta}-u$.

Choosing the arbitrary input $q \in L^{2}(0,1)$ in the adjoint problem (119) as $q(x):=(u(T)-\tilde{u})$, we deduce from the integral relationship (118) that

$$
\int_{0}^{1}(u(T)-\tilde{u}) \delta u(T) d x=\int_{0}^{T} \int_{0}^{1} \delta h v d x d t
$$


Using the integral equality (121) in the increment formula (120), we deduce that

$$
\begin{aligned}
\delta J(h)= & \int_{0}^{T} \int_{0}^{1} \delta h v d x d t \\
& +\varepsilon \int_{0}^{T} \int_{0}^{1}\left(h-h_{0}\right) \delta h d x d t \\
& +\frac{1}{2}\|\delta u(T)\|_{L^{2}(0,1)}^{2}+\frac{\varepsilon}{2}\|\delta h\|_{L^{2}(Q)}^{2} .
\end{aligned}
$$

To obtain the Fréchet differential of $\delta J(h)$, by definition, we need to show that

$$
\delta J(h)=\left\langle v+\varepsilon\left(h-h_{0}\right), \delta h\right\rangle_{L^{2}(\mathrm{Q})}+\mathcal{O}\left(\|\delta h\|_{L^{2}(\mathrm{Q})}^{2}\right) .
$$

To this aim, observe that if we substitute $t$ by $T$ in (111) it follows that

$$
\|\delta u(T)\|_{L^{2}(0,1)}^{2} \leq e^{T}\|\delta h\|_{L^{2}(Q)}^{2} .
$$

i.e., the second right-hand side integral of (122) is of the order $\mathcal{O}\left(\|\delta h\|_{L^{2}(\mathrm{Q})}^{2}\right)$. So, since the last two integrals in (122) are of the order $\mathcal{O}\left(\|\delta h\|_{L^{2}(\mathrm{Q})}^{2}\right)$, we deduce that the cost functional $J(h)$ is Frćhet-differentiable, with Fréchet differential:

$$
J^{\prime}(h)=v+\varepsilon\left(h-h_{0}\right), \quad \forall h \in \mathcal{U} .
$$

Proof of Lemma 22. The function $\delta u(t)=u^{\delta}(t)-u(t)$ solves the initial-boundary value problem

$$
\begin{aligned}
\partial_{t} \delta u & =\partial_{x}\left(a \partial_{x} \delta u\right)+\frac{\lambda}{b} \delta u+\delta h, \quad(t, x) \in Q, \\
\delta u(0) & =\delta u(1)=0, \quad t \in(0, T), \\
\delta u(0, x) & =0, \quad x \in(0,1) .
\end{aligned}
$$

Multiply both sides of the parabolic equation (126) by $v$, integrate on $Q$, and then apply the integration by parts formula on both sides multiple times. Then, taking into account the initial and boundary conditions in problem (126), we obtain the following identity:

$$
\begin{aligned}
\int_{0}^{1} \delta u(T) v(T) d x-\int_{0}^{T} \int_{0}^{1} \delta u v_{t} d x d t \\
=\int_{0}^{T} \int_{0}^{1}\left(\partial_{x}\left(a \partial_{x} \delta u\right)+\frac{\lambda}{b} \delta u+\delta h\right) v d x d t \\
=\int_{0}^{T} \int_{0}^{1}\left(\left(a v_{x}\right)_{x}+\frac{\lambda}{b} v\right) \delta u d x d t \\
\quad+\int_{0}^{T} \int_{0}^{1} \delta h v d x d t .
\end{aligned}
$$

Since $v(T)=q(x)$, thanks to (119) and (127), we arrive at

$$
\begin{gathered}
\int_{0}^{1} \delta u(T) q(x) d x=\int_{0}^{1} \delta u(T) v(T) d x \\
=\int_{0}^{T} \int_{0}^{1}\left(v_{t}+\left(a v_{x}\right)_{x}+\frac{\lambda}{b} v\right) \delta u d x d t \\
\quad+\int_{0}^{T} \int_{0}^{1} \delta h v d x d t=\int_{0}^{T} \int_{0}^{1} \delta h v d x d t
\end{gathered}
$$

This ends the proof.

4.2. Lipschitz Continuity of the Gradient and the Monotone Iteration Scheme. As emphasized in [23, Chapter 3], any gradient method for the minimization problem (104) requires an estimation of the iteration parameter $t_{n}>0$ in the iteration process

$$
h^{n+1}=h^{n}-t_{n} J^{\prime}\left(h^{n}\right), \quad n=0,1,2, \ldots
$$

where $h^{0} \in \mathcal{U}$ is a given initial iteration. The choice of $t_{n}$ defines different gradient methods, although in many situation the estimation of this parameter is a difficult problem. However, in the case of Lipschitz continuity of the gradient $J^{\prime}(h)$ the parameter $t_{n}$ can be estimated via the Lipschitz constant as follows (see [18]):

$$
0<\delta_{0} \leq t_{n} \leq \frac{2}{\left(L+2 \delta_{1}\right)}
$$

where $\delta_{0}, \delta_{1}>0$ are arbitrary parameters.

Now we will prove the Lipschitz continuity of the Fréchet gradient.

Theorem 23. Let $h, \delta h \in \mathcal{U}$. Then the Fréchet gradient (116) of the Tikhonov functional is Lipschitz continuous, i.e.,

$$
\left\|J^{\prime}\left(h^{\delta}\right)-J^{\prime}(h)\right\|_{L^{2}(\mathrm{Q})} \leq L\|\delta h\|_{L^{2}(\mathrm{Q})}, \quad \forall h, \delta h \in \mathcal{U},
$$

where the Lipschitz constant $L>0$ is defined via the parameters $T$ and $\varepsilon$ as follows:

$$
L=\sqrt{2\left(T e^{T}+\varepsilon^{2}\right)}
$$

Proof. Let the functions $v(t, x ; h)$ and $v\left(t, x ; h^{\delta}\right)$ be the solutions to the adjoint problem (119) with $q(x)=u(T, x ; h)-\tilde{u}$ and $q(x)=u\left(T, x ; h^{\delta}\right)-\tilde{u}$, respectively. We have

$$
\begin{gathered}
\left\|J^{\prime}\left(h^{\delta}\right)-J^{\prime}(h)\right\|_{L^{2}(\mathrm{Q})}^{2}=\int_{0}^{T} \int_{0}^{1}|\delta v+\varepsilon \delta h|^{2} d x d t \\
\quad \leq 2 \int_{0}^{T} \int_{0}^{1}|\delta v|^{2} d x d t+2 \varepsilon^{2}\|\delta h\|_{L^{2}(\mathrm{Q})}^{2},
\end{gathered}
$$


where $\delta v=\delta v(t, x ; h):=v\left(t, x ; h^{\delta}\right)-v(t, x ; h)$ is the solution of the backward parabolic problem

$$
\begin{aligned}
\partial_{t} \delta v+\partial_{x}\left(a \partial_{x} \delta v\right)+\frac{\lambda}{b} \delta v & =0, \quad(t, x) \in Q, \\
\delta v(0) & =\delta v(1)=0, \quad t \in(0, T), \\
\delta v(T, x) & =\delta u(T, x ; h),
\end{aligned}
$$$$
x \in(0,1)
$$

Multiplying (134) by $\delta v$, integrating over $(0,1)$, and using the boundary conditions, we get

$$
\begin{aligned}
& \frac{1}{2} \frac{d}{d t} \int_{0}^{1} \delta v(t, x ; h)^{2} d x \\
& \quad=\int_{0}^{1}\left(a\left(\partial_{x} \delta v(t, x ; h)\right)^{2}-\frac{\lambda}{b} \delta v(t, x ; h)^{2}\right) d x .
\end{aligned}
$$

Using again (15), we have

$$
\frac{1}{2} \frac{d}{d t} \int_{0}^{1} \delta v(t, x ; h)^{2} d x \geq \Lambda \int_{0}^{1} a\left(\partial_{x} \delta v(t, x ; h)\right)^{2} d x .
$$

Let

$$
\mathscr{F}(t):=\int_{0}^{1} \delta v(t, x ; h)^{2} d x .
$$

Since $\mathscr{F}^{\prime}(t) \geq 0, \mathscr{F}$ is increasing on $(0, T]$, and thus

$$
\mathscr{F}(t) \leq \mathscr{F}(T), \quad \forall t \in(0, T] .
$$

By (138), we obtain that

$$
\begin{aligned}
\int_{0}^{1} \delta v(t, x ; h)^{2} d x & \leq \int_{0}^{1} \delta v(T, x ; h)^{2} d x \\
& =\int_{0}^{1} \delta u(T, x ; h)^{2} d x .
\end{aligned}
$$

Finally, by (111) it follows that

$$
\int_{0}^{1} \delta v(t, x ; h)^{2} d x \leq e^{T}\|\delta h\|_{L^{2}(Q)}^{2} .
$$

Combining (133) and (140), we get the inequality (131).

An important application of this theorem is the following lemma (see [23, Lemmas 3.4.4 and 3.4.5]).

Lemma 24. Let the Fréchet gradient of the Tikhonov functional (104) defined on the set of admissible source terms $\mathcal{U}$ be Lipschitz continuous with the Lipschitz constant $L>0$. Denote by $\left\{h^{n}\right\} \subset \mathcal{U}$ the sequence of iterations obtained by the Landweber iteration algorithm (129). If the relaxation parameter $t_{n} \in(0,2 / L)$, then the following statements hold:

(i) $\left\{J\left(h^{n}\right)\right\}$ is a monotone decreasing sequence;

(ii) $\lim _{n \rightarrow \infty}\left\|J^{\prime}\left(h^{n}\right)\right\|_{L^{2}(\mathrm{Q})}=0$;

(iii) for the rate of convergence of the sequence $\left\{J\left(h^{n}\right)\right\}$, the following estimate holds:

$$
\begin{aligned}
0 \leq & J\left(h^{n}\right)-J_{\star} \leq 2 \operatorname{Ldn}^{-1}, \quad d>0, n=1,2,3, \ldots, \\
& \text { where } J_{\star}:=\inf _{h \in \mathcal{U}} J(h) .
\end{aligned}
$$

\section{Conclusion}

In this paper, we have considered an inverse source problem for a class of degenerate and singular parabolic equations. Based on Carleman estimates, global Lipschitz stability result is proved. Then, the identification of the source term is formulated as a minimization problem combining the output least squares and the Tikhonov regularization. It is proved that the Fréchet derivative of the cost functional can be formulated via the solution of the adjoint parabolic problem. Lipschitz continuity of the gradient functional was also proved, which implies the monotonicity of the numerical sequence of iterations obtained by the Landweber iteration algorithm. Some applications with numerical implementations are in progress.

\section{Data Availability}

No data were used to support this study.

\section{Conflicts of Interest}

The authors declare that there are no conflicts of interest regarding the publication of the paper.

\section{References}

[1] J. Vancostenoble, "Lipschitz stability in inverse source problems for singular parabolic equations," Communications in Partial Differential Equations, vol. 36, no. 8, pp. 1287-1317, 2011.

[2] O. Y. Imanuvilov and M. Yamamoto, "Lipschitz stability in inverse parabolic problems by the Carleman estimate," Inverse Problems, vol. 14, no. 5, pp. 1229-1245, 1998.

[3] P. Cannarsa, J. Tort, and M. Yamamoto, "Determination of source terms in a degenerate parabolic equation," Inverse Problems, vol. 26, no. 10, 105003, 20 pages, 2010.

[4] J.-P. Puel and M. Yamamoto, "On a global estimate in a linear inverse hyperbolic problem," Inverse Problems, vol. 12, no. 6, pp. 995-1002, 1996.

[5] A. L. Bukhgeim and M. V. Klibanov, "Global uniqueness of a class of multidimensional inverse problems," Soviet Mathematics-Doklady, vol. 24, pp. 244-247, 1981.

[6] A. V. Fursikov and O. Y. Imanuvilov, Controllability of Evolution Equations, vol. 34 of Lecture Notes Series, Seoul National University, Research Institute of Mathematics, Global Analysis Research Center, Seoul, South Korea, 1996.

[7] I. Boutaayamou, G. Fragnelli, and L. Maniar, "Carleman estimates for parabolic equations with interior degeneracy and Neumann boundary conditions," Journal d'Analyse Mathématique, vol. 135, no. 1, pp. 1-35, 2018.

[8] P. Cannarsa, P. Martinez, and J. Vancostenoble, "Carleman estimates for a class of degenerate parabolic operators," SIAM Journal on Control and Optimization, vol. 47, no. 1, pp. 1-19, 2008.

[9] G. Fragnelli and D. Mugnai, "Carleman estimates and observability inequalities for parabolic equations with interior degeneracy," Advances in Nonlinear Analysis, vol. 2, no. 4, pp. 339-378, 2013.

[10] I. Boutaayamou, G. Fragnelli, and L. Maniar, "Inverse problems for parabolic equations with interior degeneracy and Neumann 
boundary conditions," Journal of Inverse and ILL-Posed Problems, vol. 24, no. 3, pp. 275-292, 2016.

[11] I. Boutaayamou, G. Fragnelli, and L. Maniar, "Lipschitz stability for linear parabolic systems with interior degeneracy," Electronic Journal of Differential Equations, No. 167, 26 pages, 2014.

[12] J. Tort, "Determination of source terms in a degenerate parabolic equation from a locally distributed observation," Comptes Rendus MathÉmatique. AcadÉMIe des Sciences. Paris, vol. 348, no. 23-24, pp. 1287-1291, 2010.

[13] Z.-C. Deng, K. Qian, X.-B. Rao, L. Yang, and G.-W. Luo, "An inverse problem of identifying the source coefficient in a degenerate heat equation," Inverse Problems in Science and Engineering, vol. 23, no. 3, pp. 498-517, 2015.

[14] X.-B. Rao, Y.-X. Wang, K. Qian, Z.-C. Deng, and L. Yang, "Numerical simulation for an inverse source problem in a degenerate parabolic equation," Applied Mathematical Modelling: Simulation and Computation for Engineering and Environmental Systems, vol. 39, no. 23-24, pp. 7537-7553, 2015.

[15] K. Atifi, Y. Balouki, El-H. Essoufi, and B. Khouiti, "Identifying Initial Condition in Degenerate Parabolic Equation with Singular Potential," International Journal of Differential Equations, vol. 2017, Article ID 1467049, 17 pages, 2017.

[16] G. Fragnelli and D. Mugnai, "Carleman estimates for singular parabolic equations with interior degeneracy and non smooth coefficients," Advances in Nonlinear Analysis, 2016.

[17] P. Cannarsa, P. Martinez, and J. Vancostenoble, "Global Carleman estimates for degenerate parabolic operators with applications," Memoirs of the American Mathematical Society, vol. 239, no. 1133, ix+209 pages, 2016.

[18] A. Hasanov, "Simultaneous determination of source terms in a linear parabolic problem from the final overdetermination: weak solution approach," Journal of Mathematical Analysis and Applications, vol. 330, no. 2, pp. 766-779, 2007.

[19] A. Bensoussan, G. da Prato, M. Delfour, and S. K. Mitter, Representation and Control of Infinite Dimensional Systems, Birkhäuser, 2nd edition, 2007.

[20] G. Fragnelli and D. Mugnai, "Carleman estimates, observability inequalities and null controllability for interior degenerate non smooth parabolic equations," Memoirs of the American Mathematical Society, vol. 242, p. 1164, 2016.

[21] A. Hasanov, P. DuChateau, and B. Pektas, "An adjoint problem approach and coarse-fine mesh method for identification of the diffusion coefficient in a linear parabolic equation," Journal of Inverse and ILL-Posed Problems, vol. 14, no. 5, pp. 435-463, 2006.

[22] Y. H. Ou, A. Hasanov, and Z. H. Liu, "Inverse coefficient problems for nonlinear parabolic differential equations," Acta Mathematica Sinica, vol. 24, no. 10, pp. 1617-1624, 2008.

[23] A. Hasanov and V. G. Romanov, Introduction to inverse problems for differential equations, Springer, Heidelberg, Germany, 2017. 


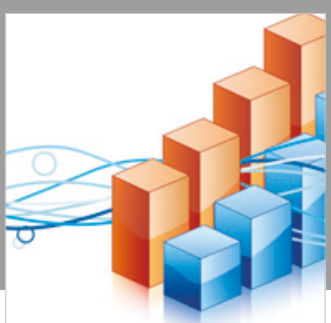

Advances in

Operations Research

\section{-n-m}
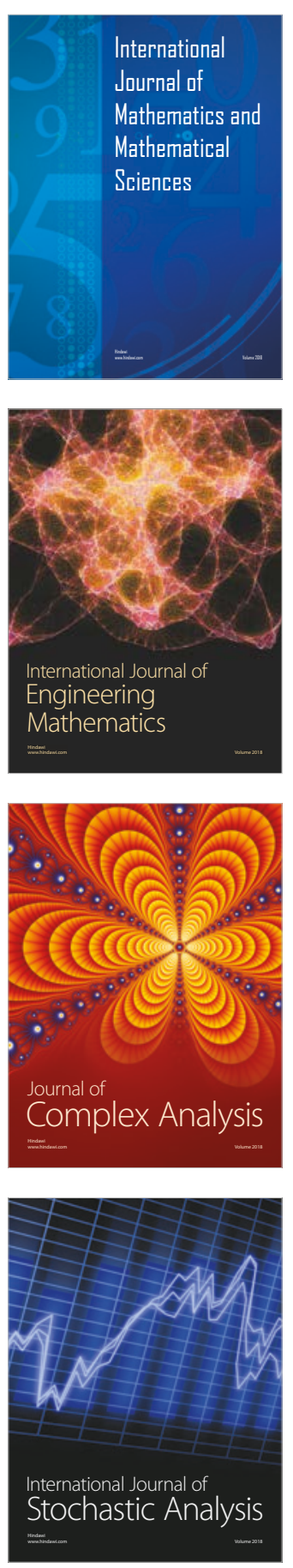
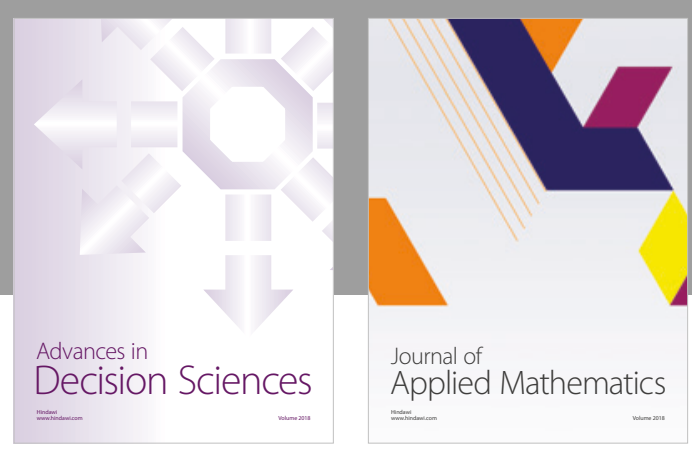

Journal of

Applied Mathematics
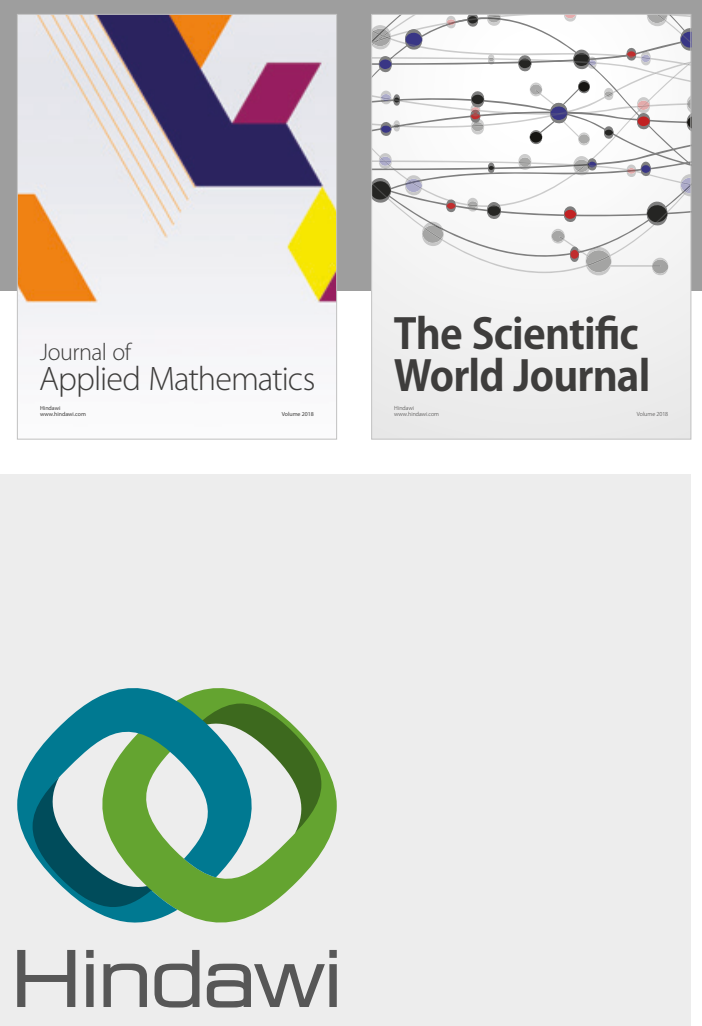

Submit your manuscripts at

www.hindawi.com

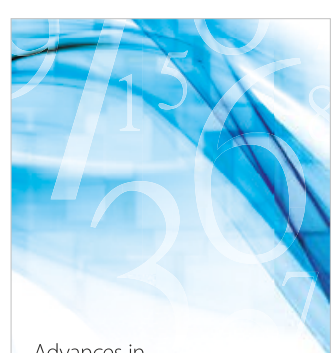

Advances in
Numerical Analysis
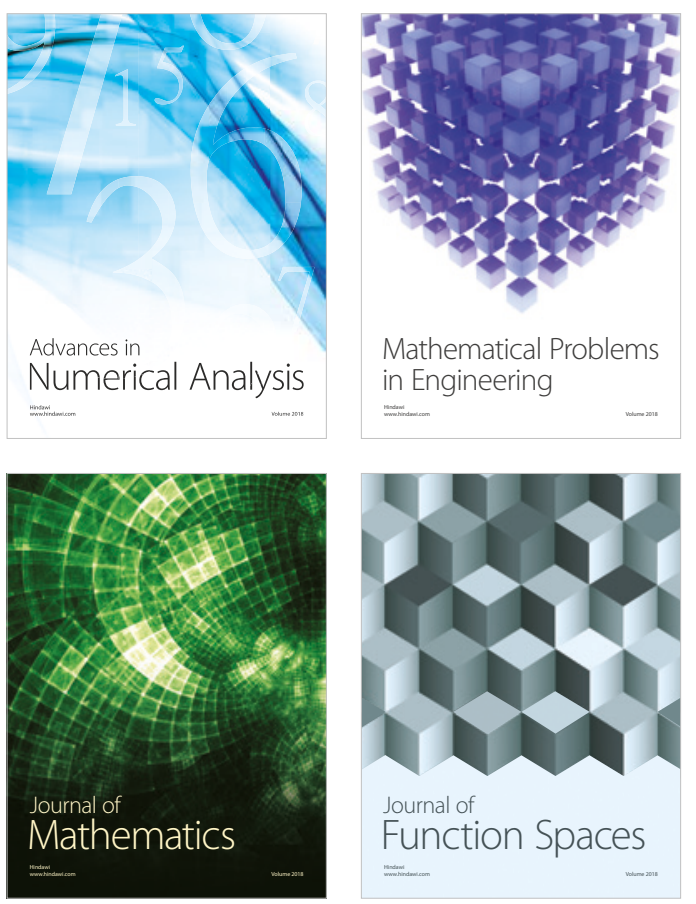

Mathematical Problems in Engineering

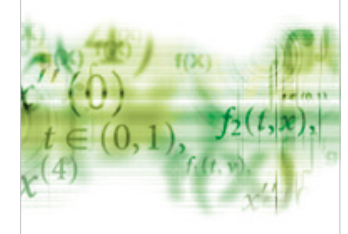

International Journal of

Differential Equations

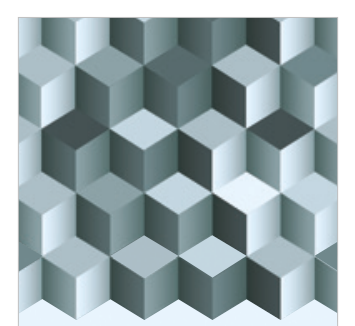

Journal of

Function Spaces

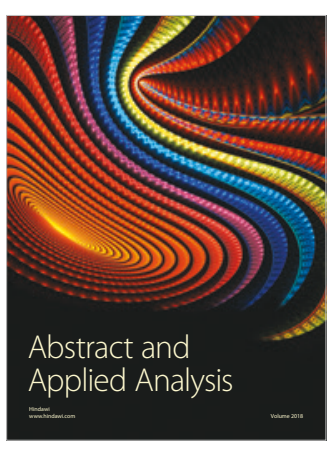

The Scientific

World Journal

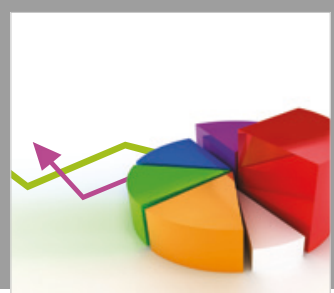

Journal of

Probability and Statistics
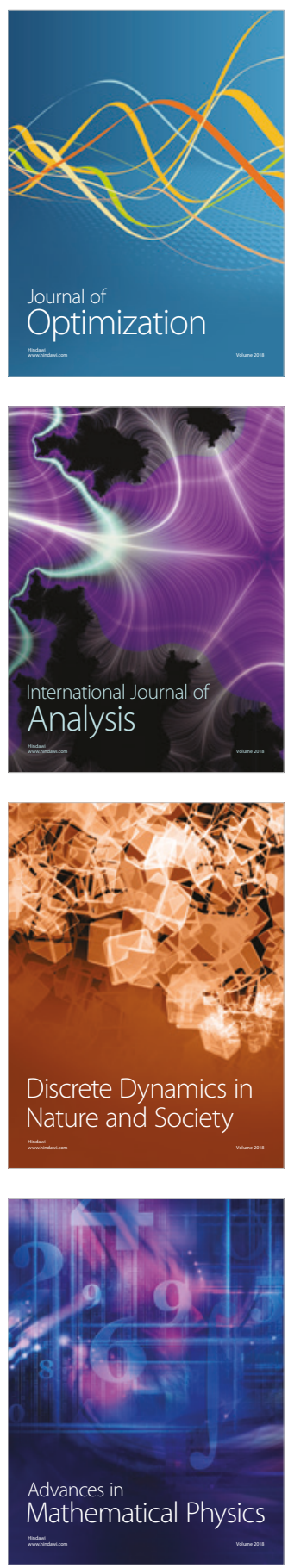